\title{
Neutron applications developing at compact accelerator-driven neutron sources
}

\author{
Yoshiaki Kiyanagi ${ }^{1,2}$
}

\begin{abstract}
Neutrons have been used in a wide field of applications by using various neutron sources. Material science is one of the widest research fields. The activity is supported by nuclear research reactors and high-intensity spallation neutron sources based on a high-intensity proton accelerator. However, it is desired to perform a measurement when researchers want to do and to perform adventuresome experiments that have not yet confirmed its importance. Furthermore, trial and error measurements are necessary to improve a measurement method. Compact accelerator-driven neutron sources are suitable for such usage and in some cases can complement the measurement at a large facility. The use of the compact neutron source has sometimes led to new methods. Other than material science, a new application of soft error acceleration test has been performed at the compact accelerator-driven neutron sources. Another neutron application is radiation therapy called as boron neutron capture therapy. In this field, nuclear reactor neutron sources have been used but many of them shut down. It was desired to construct the BNCT facility near a hospital. Therefore, BNCT facilities based on the compact accelerator have been constructed in the world. Here, the neutron sources and new methods and applications developing at compact accelerator-driven neutron sources are introduced.
\end{abstract}

Keywords: Compact accelerator-driven neutron source, Neutron production reaction, Neutron intermediate-angle scattering, Pulsed neutron imaging, Soft error test, Boron neutron capture therapy

\section{Background}

Neutron sources are shifting from research nuclear reactors to accelerator-driven neutron sources. Large neutron sources based on a high-energy proton accelerator have been constructed for neutron science applications. On the other hand, compact accelerator-driven neutron sources also have been constructed, under construction and planning. The compact accelerator-driven neutron sources are used for various applications. New methods were developed in neutron scattering for material science; soft error test of semiconductor device has been executed and boron neutron capture therapy is being promoted by using neutron sources based on compact

Correspondence: kiyanagi@phi.phys.nagoya-u.ac.jp

${ }^{1}$ Nagoya University, Nagoya, Japan

${ }^{2}$ Present Address: Japan Neutron Optics Inc., Gamagori, Japan accelerators. These recent activities are explained in this review paper as well as neutron sources in the world.

\section{Introduction}

Neutrons have been used for neutron science, medical treatment, and irradiation. Research nuclear reactors have been used as neutron sources for a long time. However, many reactor neutron sources shut down and some of the existing ones have a plan to shut down in the near future $[1,2]$. On the other hand, accelerator-driven neutron sources based on a spallation reaction have been constructed in the world to supply high-intensity neutron beams $[1,3]$ to support and develop neutron activities. There are four spallation neutron sources under operation in the world: SNS and LANSCE in the USA, JSNS in Japan, ISIS in the UK, and CSNS in China. In 
addition to them, ESS is under construction in Sweden. Furthermore, compact accelerator-driven neutron sources (CANSs) have also been constructed for science and radiation treatment [2, 3]. In material researches and industrial applications, it is better for researchers to have a chance to measure a sample before an experiment at a large facility. The CANS has better characteristics than high-intensity sources although the neutron intensity is much lower than and the resolution is not high as the high-intensity ones. Usually, there is no review system for an experimental proposal, which allows the researchers to perform a venturous measurement. The experiment will be done on-demand like, namely, the researcher can perform the experiment not waiting for a long time as in the high-intensity sources. Not only instrument-depending experiments like the diffractometer but also a new type of experiment can be executed easier. Such characteristics sometimes led to the development of new methods [4-6].

On the other hand, neutrons have been also used for medical treatment by using reactor neutron sources for a long time. Boron neutron capture therapy (BNCT) has been performed at reactor sources in the world and its usefulness has been proved [7]. However, the reactor sources were not convenient for treatment since they could not be constructed near or in a hospital. Therefore, it has been desired to construct accelerator-driven neutron sources for BNCT. Recent accelerator development to attain a power around few tens $\mathrm{kW}$ supported this trend, and various BNCT facilities based on the accelerator were constructed, are under construction and planned in the world. In 2020, a system obtained medical device approval for manufacturing and sales of accelerator-based BNCT system and the dose calculation program in Japan (https://www.shi.co.jp/english/info/201 9/6kgpsq0000002ji0.html). It is the world's first medical device of BNCT and two BNCT hospitals have started treatment.

One of the other applications recently developed is a soft error acceleration test that makes it possible to check the effectiveness of methods to reduce the soft error and estimate an acceleration rate [8]. The soft error is becoming more or more important since IT equipment prevails to various fields and increases its total number in the world. The soft error is not a fatal error of IC that is called as a hard error since it can be recovered by resetting the system. This is a bit error caused by the extra charge produced with radiations. Neutron is a major contributor at Earth's surface. So far, the spallation neutron sources have been used since they can well simulate the spectrum at Earth's surface (https://permalink.lanl.gov/object/tr?what=info:lanlrepo/lareport/LA-UR-05-8767). However, the use of a CANS to evaluate the error rate is useful and a new idea since it was thought that a neutron spectrum similar to the natural terrestrial one was necessary for such a measurement. There are several merits to perform the experiment at compact neutron sources since it is easier to access the facility and to manage experiments although their neutron spectra are different from the natural terrestrial one.

Here, neutron intensity and energy range required for neutron applications, accelerator-based neutron sources, neutron applications recently developing at CANSs: small and medium angle neutron scattering, neutron imaging, soft error test, and BNCT application, are introduced.

\section{Neutron intensity and feasible applications}

There are various neutron applications. In Fig. 1, an energy and intensity range is roughly indicated for each measurement or application although it is just for reference. Here, the intensity means the produced neutron intensity at a neutron production area. The intensity limits indicated are determined by not only statistic reason of the measurements but also economical or physical condition of each application. At low neutron intensity, moisture measuring [9], water level gauge, and oil prospecting $[10,11]$ have been executed by using high-energy neutrons emitted from a radioisotope neutron source or a neutron tube. In these experiments, a portable or easy to bring neutron source is used, and due to this reason, the intensity necessarily becomes low.

At higher intensity, various neutron scattering experiments are performed. Neutron scattering experiments are, as prime examples, imaging, diffraction, small-angle scattering, reflectometer, and quasi and inelastic scattering [1]. Intensity ranges shown in Fig. 1 are required for the accelerator-driven neutron source. Higher intensity is better for all experiments since usually statistics and resolution become better or the amount of the sample required for measurement becomes small with increasing the intensity. Many of the measurements can be done from an intensity about $10^{12} \mathrm{n} / \mathrm{s}$ but quasi-elastic and inelastic scattering experiments need much higher intensity since they analyze the scattered neutron energy. The energy range required for each experiment changes largely. However, many of them are in a range of cold $(\sim 0.1 \mathrm{meV}$ to $5 \mathrm{meV})$ and thermal neutrons $(\sim 5 \mathrm{meV}$ to $\sim 1 \mathrm{eV})$. Imaging can be performed in a very wide energy range, since neutron interaction cross section depends on energy and so we can obtain a different contrast image with changing the energy. Prompt $\gamma$-ray analysis is a useful method for microanalysis and it uses cold to thermal neutrons. Fundamental physics occupy two energy regions. One is very low energy from ultra-cold to cold and the other is a high-energy region of sub $\mathrm{MeV}$ to $\mathrm{MeV}$. 


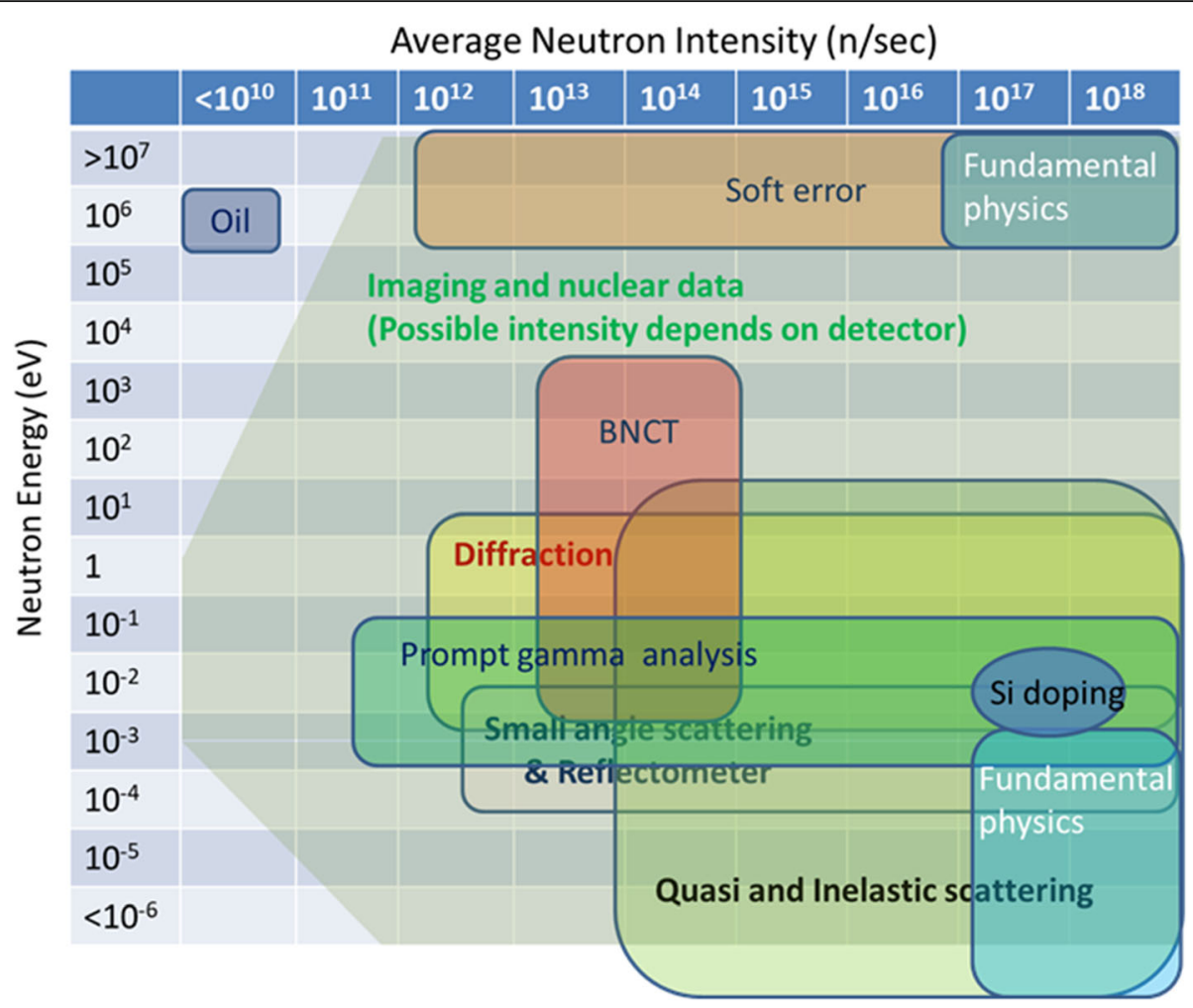

Fig. 1 Neutron intensity and energy range for various neutron applications and measurements

Si doping is one of the important applications at reactor neutron sources. This is a method to create impurity homogeneously in $\mathrm{Si}$ block using thermal neutron penetrating power, in which ${ }^{30} \mathrm{Si}(\mathrm{n}, \gamma){ }^{31} \mathrm{Si}$ and ${ }^{31} \mathrm{Si} \rightarrow{ }^{31} \mathrm{P}$ reaction process occurs. For the neutron absorption reaction, thermal neutrons are effective. Radioisotope production is another useful application.

The soft error acceleration test is one of the irradiation applications. The energy range used is mainly over few $\mathrm{MeV}$ since major nuclear reactions occur in this energy range for the Si nucleus, a major element in a IT device. However, even in a much lower energy region, namely, the thermal neutron region, the soft errors were observed depended on devices.

For a BNCT neutron source, higher intensity is preferable but higher intensity requires higher accelerator power, namely, beam current times particle energy. It means that construction cost becomes expensive since a high intensity neutron source requires not only an expensive accelerator but also a thick shielding. Engineering restrictions existed to increase the power have been solved recently. The neutron intensity produced at BNCT sources is about $10^{13}-10^{14} \mathrm{n} / \mathrm{sec}$ order that corresponds to about few tens $\mathrm{kW}$ beam power.

Other applications such as neutron device development can be done at a low-intensity facility. This kind of development is useful for high-intensity neutron sources to save their machine time and the result will contribute to improve the instruments at the high-intensity neutron sources.

The measurements that can be performed at CANSs are the ones that the intensity limit is less than about $10^{12} \mathrm{n} / \mathrm{s}$. Anyway, it should be noted that the indicated energy and intensity ranges are just for guide and not rigorous.

\section{Neutron sources based on accelerators}

Neutron science and industrial applications are actively performed at various neutron sources. Many of the research reactor neutron sources were shut down and recently, as examples, ORPHEE in France and BER-II in Germany were closed in 2019. Instead of the reactor source, accelerator-driven neutron sources have been proposed in Europe [12, 13].

There are various neutron production nuclear reactions. Radioisotope neutron sources like ${ }^{252} \mathrm{C}_{\mathrm{f}}, \mathrm{Am}-\mathrm{Be}$, etc. are used as low-intensity and easy-to-handle neutron sources. ${ }^{252} \mathrm{C}_{\mathrm{f}}$ are sometimes used for neutron imaging. One of the more intense neutron sources is neutron tubes using $\mathrm{d}-\mathrm{d}$ or $\mathrm{d}-\mathrm{t}$ nuclear reactions [14]. The $\mathrm{d}-\mathrm{t}$ reaction is ${ }^{2} \mathrm{H}+{ }^{3} \mathrm{H} \rightarrow{ }^{4} \mathrm{He}+\mathrm{n}+17.59 \mathrm{MeV}$, and $\mathrm{d}-\mathrm{d}$ is ${ }^{2} \mathrm{H}+{ }^{2} \mathrm{H} \rightarrow{ }^{3} \mathrm{He}+\mathrm{n}+3.27 \mathrm{MeV}$. The $\mathrm{d}-\mathrm{t}$ neutron tube produces about 100 times higher intensity than the $\mathrm{d}-\mathrm{d}$ source. However, tritium handling causes major 
difficulty to use in a laboratory due to its severe regulation. Therefore, the $\mathrm{d}-\mathrm{d}$ neutron tube is used and applied to various applications, to cite a case, imaging. However, the intensity is at most less than about $10^{12} \mathrm{n} / \mathrm{s}$ and not enough for many of the neutron applications mentioned before.

To obtain much higher neutron intensity, we need to use accelerators. The most effective reaction is spallation. As shown in Fig. 2a, the spallation reaction is caused by a high-energy proton injection usually to high atomic number materials since the number of neutrons is larger in high atomic number materials. The highenergy proton induces an intra-nuclear cascade in a nucleus. The proton directly sputters particles of neutron, proton, pion, etc. and then the nucleus is left at an

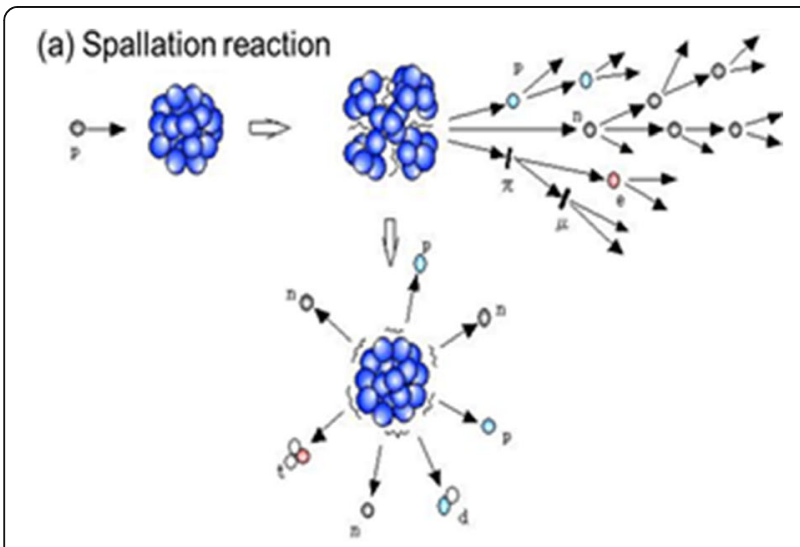

(b) Photo-neutron reaction

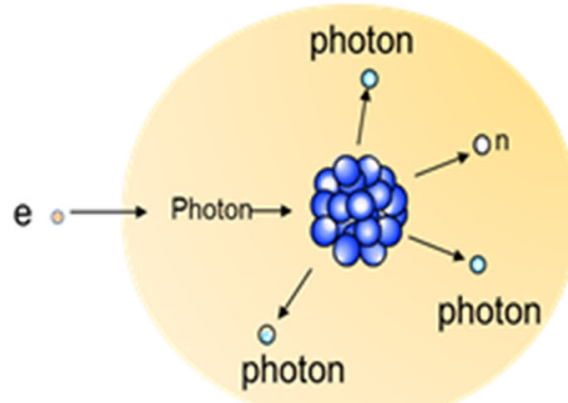

(c) p-Be reaction

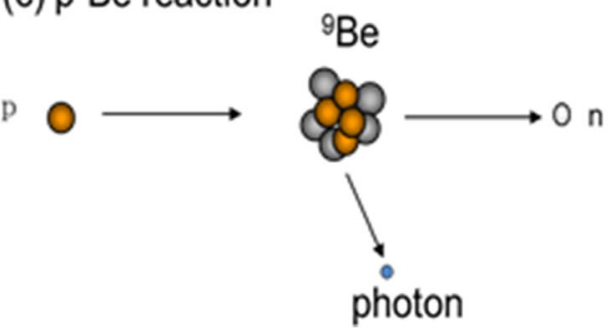

Fig. 2 Conceptual diagram of neutron production reactions. a Spallation reaction, $\mathbf{b}$ photo-neutron reaction, and $\mathbf{c} \mathrm{p}$-Be reaction excited state. Neutrons are released from the nucleus through an evaporation process of the excited nucleus. About $90 \%$ neutrons are produced by this process. A major part of the energy spectrum is similar to that of fission and a peak energy appears around $1 \mathrm{MeV}$. However, the spectrum has a gradually decreasing tail up to the energy of the incident proton. The number of neutrons is $\sim 10^{14} \mathrm{n} / \mathrm{s}$ at $1 \mathrm{~kW}$ power. This is about 100 times higher efficiency than other reactions. However, to induce the spallation reaction, high-energy protons are necessary with an energy more than about $100 \mathrm{MeV}$ and this requires a big and expensive accelerator. Therefore, only one neutron source has been constructed in each country or a wider area. High-intensity neutron sources are indispensable for the advanced application of neutron science and industrial use but CANSs other than spallation are necessary to make various neutron applications more popular. The nuclear reactions used at compact accelerators are photo-neutron, $\mathrm{p}-\mathrm{Be}$, and $\mathrm{p}-\mathrm{Li}$. As shown in Fig. 2b, the photo-neutron is a reaction caused by electrons in which an electron first interacts with an electric field in an electron shell and then emits a high-energy photon through bremsstrahlung; succeedingly, the photon injected into the nucleus excites the nucleus; and finally, a neutron is released from the nucleus through the evaporation process. Therefore, the energy spectrum has a shape of evaporation neutrons with a peak energy around $1 \mathrm{MeV}$ like spallation. However, in this reaction, the energy spectrum does not have a tail that the spallation spectrum has. The neutron yield is about $10^{12} \mathrm{n} / \mathrm{s}$ at $1 \mathrm{~kW}$. Recently, compact proton accelerators have been constructed for the neutron beam experiment and the BNCT facility. The nuclear reactions are as follows:

$$
\begin{aligned}
& { }^{7} \mathrm{Li}+\mathrm{p} \rightarrow{ }^{7} \mathrm{Be}+\mathrm{n}-1.65 \mathrm{MeV} \\
& { }^{9} \mathrm{Be}+\mathrm{p} \rightarrow{ }^{9} \mathrm{~B}+\mathrm{n}-1.85 \mathrm{MeV}
\end{aligned}
$$

The $\mathrm{p}$-Be reaction is indicated in Fig. $2 \mathrm{c}$ and the scheme of $\mathrm{p}-\mathrm{Li}$ reaction is very similar to this. Their threshold energies are $1.88 \mathrm{MeV}$ for $\mathrm{Li}$ and $2.06 \mathrm{MeV}$ for Be. Many of the neutron sources use these reactions. For the BNCT source, the $\mathrm{p}-\mathrm{Li}$ reaction is becoming more popular in the world since it produces high-intensity neutrons at lower proton energy than $\mathrm{Be}$, which leads to low-energy neutrons. The low-energy neutron is efficiently moderated to required energies in a moderator. It should be noted that ${ }^{7} \mathrm{Be}$ is a radioactive nucleus with a half-life of 53.12 days. The number is the same as the neutron number but only $10.4 \%$ of ${ }^{7} \mathrm{Be}$ decay emitting $478 \mathrm{keV} \gamma$-ray. In the case of the $\mathrm{p}$-Be reaction, a 
reaction channel producing ${ }^{7} \mathrm{Be}$ and tritium opens over about $13 \mathrm{MeV}$.

Other candidates are d-Li and d-Be reactions.

$$
{ }^{7} \mathrm{Li}+\mathrm{d} \rightarrow 2^{4} \mathrm{He}+\mathrm{n}+15.12 \mathrm{MeV}
$$

$$
{ }^{9} \mathrm{Be}+\mathrm{d} \rightarrow{ }^{10} \mathrm{~B}+\mathrm{n}+4.36 \mathrm{MeV}
$$

They are all exothermic reactions. Therefore, a merit of the reactions is that they allow to adopt a low-energy accelerator. The $\mathrm{d}$-Be reaction has smaller fraction of high-energy neutron intensity of $\mathrm{MeV}$ order than the d$\mathrm{Li}$ one. It should be considered in the $\mathrm{d}$-Be reaction that tritium is produced by about half of the neutron number.

Figure 3 shows the neutron yield depending on the particle energy. The data about $\mathrm{d}-\mathrm{Be}$ and $\mathrm{d}-\mathrm{Li}$ are from Ref. [15], and p-Li, p-Be, and photo-neutron from Ref. [16]. The $\mathrm{d}-\mathrm{Li}$ and the $\mathrm{d}-\mathrm{Be}$ reactions can be used for low-energy accelerators. The $\mathrm{p}-\mathrm{Li}$ is next to these reactions and used around $3 \mathrm{MeV}$. The p-Be reaction needs higher energy accelerators than the $\mathrm{p}-\mathrm{Li}$ one to obtain a high-intensity neutron beam but the handling of a $\mathrm{Be}$ target is easier than a Li target. The photo-neutron reaction needs accelerators with an energy over around 20
$\mathrm{MeV}$ since the neutron production reaction has a threshold energy usually more than about $10 \mathrm{MeV}$. The electron linear accelerator has a merit to produce a short pulse beam less than about few $\mu$ sec, which enables us to measure the resonance peak in the neutron cross section.

\section{Applications at compact accelerator-driven neutron sources}

The CANS is not based on the spallation reaction and the intensity is less than about $10^{14} \sim 10^{15} \mathrm{n} / \mathrm{s}$. There are various neutron sources in this intensity range in the world. Figure 4 shows CANSs as well as spallation neutron sources in the world used for the neutron scattering experiment. The spallation neutron sources are indicated by a yellow background color. ESS is under construction and others are operating. Figure 5 shows their intensity, where two kinds of intensities are indicated at the $X$ and $Y$ axes [17]. The $X$ axis is a peak intensity that is defined as (Neutron intensity in a pulse duration)/(Duration time) and the $Y$ axis is an average intensity that is averaged over a long time range compared with each beam pulse duration. Here, a line indicating two intensities are equal (namely, steady source) is shown. Even among the accelerator sources, SINQ and NUANS are steady neutron source, not pulsed one. HUNS at Hokkaido

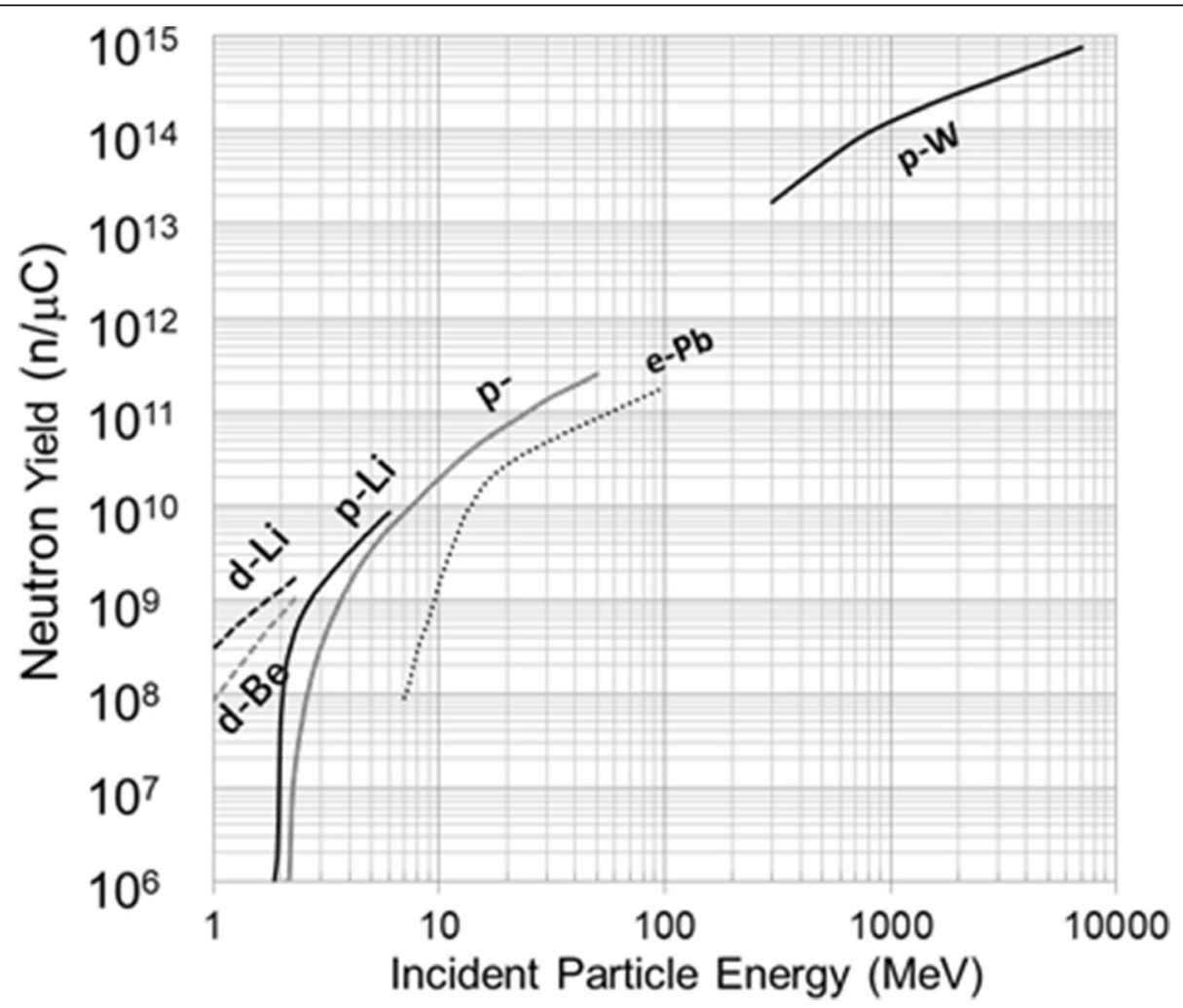

Fig. 3 Neutron yield as a function of incident particle energy 


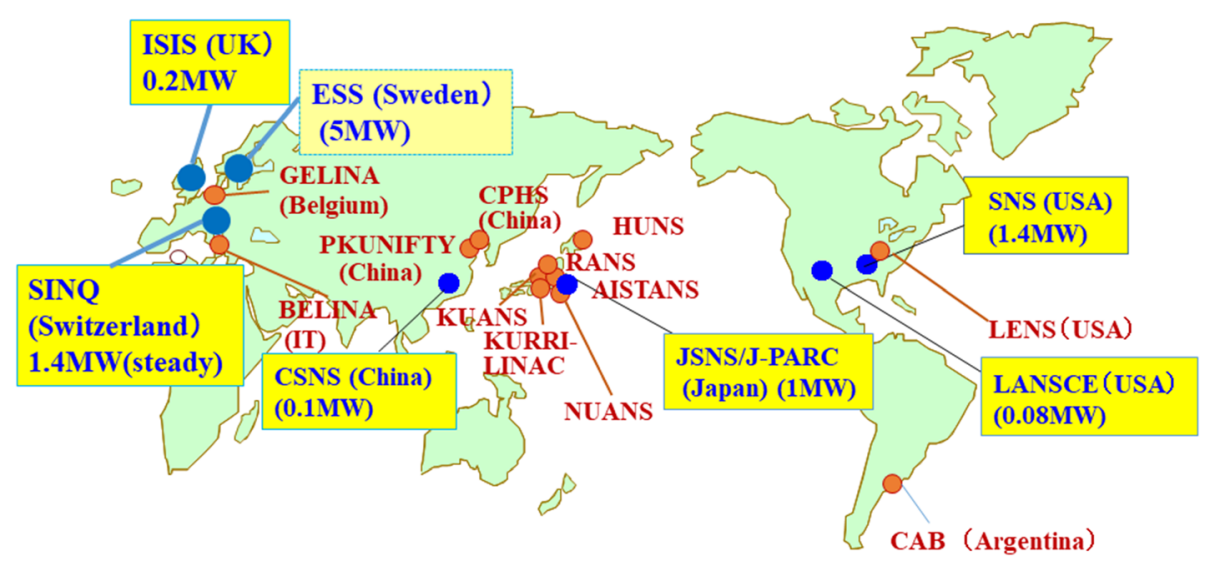

Fig. 4 Accelerator-driven neutron sources used for neutron beam experiments in the world

University has a long history as a CANS [18]. This source uses an electron linear accelerator and is applied to imaging, intermediate-angle scattering. GELINA in Belgium is also an electron accelerator based neutron source (https://ec.europa.eu/jrc/en/research-facility/ linear-electron-accelerator-facility), which are mainly used for nuclear data measurements and also for resonance imaging. LENS at Indiana University in the USA was constructed using a proton accelerator with a Be target (https://ceem.indiana.edu/lens/about-lens/index. html), which is used for small-angle neutron scattering (SANS) and spin-echo scattering, and also used to obtain data such as moderator development for SNS in the USA. A soft error test can be performed at an irradiation port. RANS at RIKEN [19], KUANS at Kyoto University in Japan, and CPHS at Tsinghua University in China [20] are based on a proton accelerator with the use of Be target. RANS is used for imaging, diffraction, and SANS, and KUANS for imaging. CPHS was planned for imaging and SANS. PKUNIFTY at Peking University in China is based on a deuteron accelerator with a Be target and used for imaging [21]. The neutron intensity of KUANS is of the order of $10^{11} \mathrm{n} / \mathrm{s}$ and others are $10^{12} \sim 10^{13} \mathrm{n} / \mathrm{s}$. Recently, an electron linac-based neutron source (AIST ANS) was constructed in Japan [22] mainly for Bragg edge transmission measurement [5].

Various applications are being performed at CANSs and all the sources execute device development such as

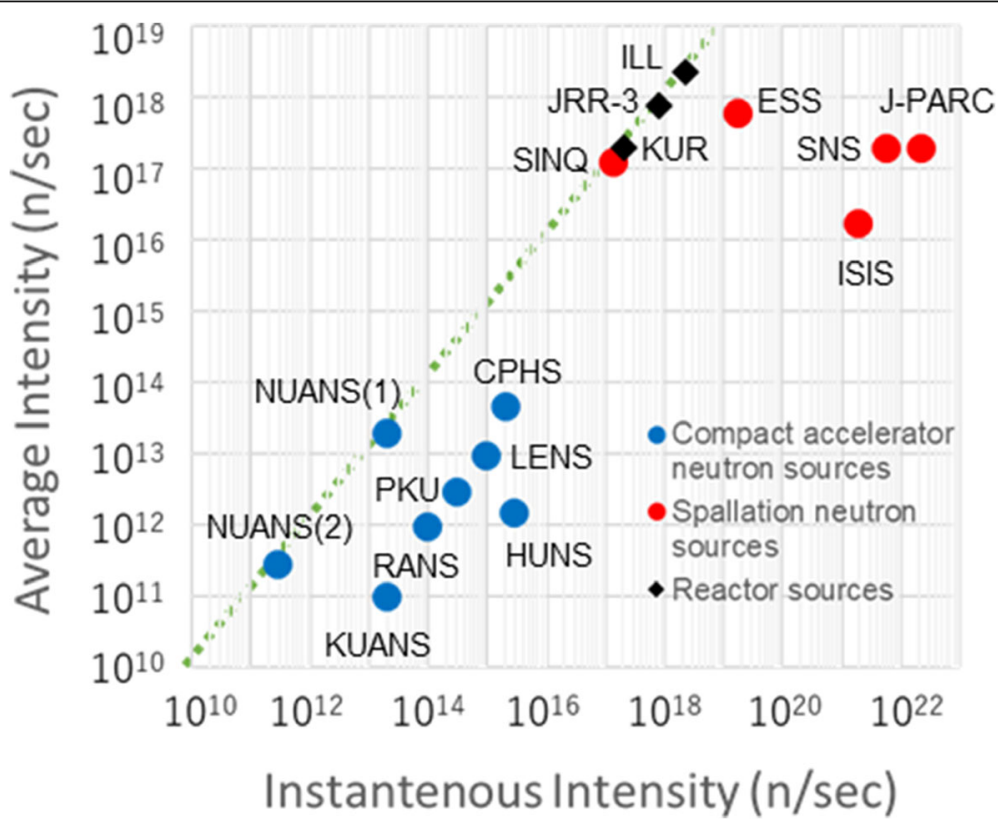

Fig. 5 Average and instantaneous neutron intensities of accelerator-driven neutron sources [17] 
detector, mirror, focusing device, etc. Here, we introduce new neutron measurements and applications developing by using CANSs.

\subsection{Intermediate-angle neutron scattering [6]}

SANS is one of the most important tools to investigate the nanostructure of materials. Polymer and biomaterials are suitable research items for SANS. On the other hand, it has been used to study metal materials. Intensity difference between the high-intensity source and CANS is more than 3 orders. Therefore, to perform the same experiment at the high-intensity source cannot be executed at CANS. However, CANS has various merits to proceed with the material research. It is required to determine a target research object and optimize the instrument according to the requirement to use the CANS effectively. Based on this consideration, an intermediateangle scattering instrument, iANS, was developed with the use of the time-of-flight method at HUNS for characterizing nanostructures embedded in a metallic matrix [6].

Major SANS systems are designed for measurement at low- $Q$ region. Here, $Q$ is the momentum transfer defined as $Q=4 \pi \sin \theta / \lambda$ ( $2 \theta$ : scattering angle, $\lambda$ : neutron wavelength). However, in metallurgy, there are many tools for characterization from the micron scale to a few ten nanometers. In contrast, the choice of characterization methods is limited when the size of heterogeneities reaches a few nanometers. Therefore, iANS was designed to observe the $Q$ range from 0.2 to 10 $\mathrm{nm}^{-1}$, a region already indicated its usefulness by laboratory SAXS [23-25]. It is advantageous that instruments at CANS can be optimized from the source to the detector for this fixed purpose.

The most important key is to optimize the $Q$ resolution. If the $Q$ resolution can be relaxed, it allows us to use a larger divergence neutron beam than that of a traditional SANS. The $Q$ resolution is defined as $\Delta Q / Q$, where $\Delta Q$ is the FWHM value in the case of a Gaussian distribution. Because the measured detector count rate $C_{\mathrm{D}}$ is roughly proportional to the fourth power of $\Delta Q$, the gained flux can be expressed as follows:

$$
\mathrm{C}_{\mathrm{D}} \propto \Delta Q^{4}
$$

Since some of the other effects are ignored for simplification, $C_{\mathrm{D}}$ will be overestimated when using this equation. If the $Q$ resolution is set to $\Delta Q / Q=30 \%$, then $\Delta Q$ $=0.06 \mathrm{~nm}^{-1}$ at $Q_{\min }=0.2 \mathrm{~nm}^{-1}$ is required for iANS optics, while $\Delta Q=0.009 \mathrm{~nm}^{-1}$ at $Q_{\min }=0.03 \mathrm{~nm}^{-1}$ is needed for regular SANS instruments. Consequently, the $C_{\mathrm{D}}$ of iANS is at most $(0.06 / 0.009)^{4}=2000$ times higher than that of a regular SANS system. Under these considerations, the optimum design was determined and the layout is shown in Fig. 6 [6]. The design parameters are $Q_{\min }=0.25 \mathrm{~nm}^{-1}, Q_{\max }=6.5 \mathrm{~nm}^{-1}$, available wavelength $=0.07-1.3 \mathrm{~nm}$, detector area $=600 \mathrm{~mm} \times 200 \mathrm{~mm}$, detector element size $=5 \mathrm{~mm} \times 12.7 \mathrm{~mm}, L_{1}$ (source to sample length) $=5500 \mathrm{~mm}, L_{2}$ (sample to detector length) $=500 \mathrm{~mm}, d_{1}$ (1st pinhole diameter: moderator size) $=100 \mathrm{~mm}$, and $d_{2}$ (sample size $)=\phi 10$ or $\phi 13 \mathrm{~mm}$.

The performance was compared with data obtained at a high-intensity reactor source and showed good coincidence in the targeted $Q$ range. As one of the examples of iANS measurements, SANS data are compared to those obtained by small-angle X-ray scattering (SAXiS) with respect to SAXS/SANS contrast variation to evaluate precipitates [26]. This is a useful method provided by in-house SANS and SAXS. The scattering intensity of the small-angle scattering is written by the following equation for neutron and X-ray.

Scattering intensity $=(\Delta \rho)^{2} \times($ Number density $) \times$ (VolumexGrain form factor) ${ }^{2}$.

The terms other than the first one are common for both radiations and determines the shape of the $Q$ dependent scattering spectrum. The first term is

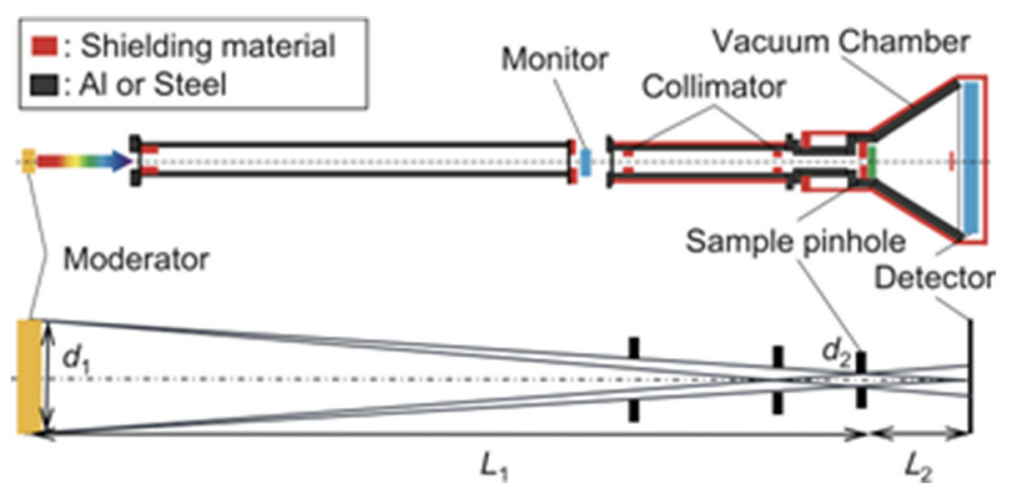

Fig. 6 Layout of an intermediate-angle scattering instrument, iANS at HUNS [6] 


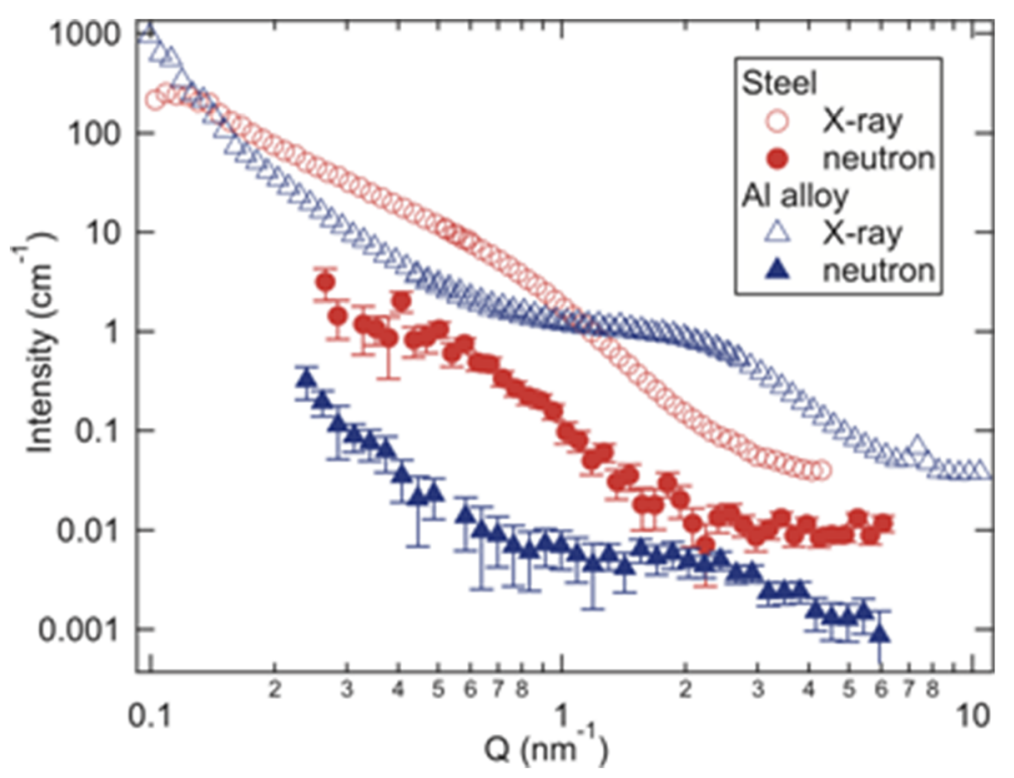

Fig. 7 Intermediate-angle scattering intensities for Ti-added steel (circles) and 700 series aluminum alloy (triangles) [6]

determined by the scattering amplitude of each radiation. The amplitude changes from element to element for the neutron but monotonically increases with the atomic number for X-ray. Therefore, we will identify precipitates by using the scattering intensity change between SANS and SAXS measurements. Figure 7 shows $\mathrm{TiC}$ precipitates in the steel and $\mathrm{MgZn}_{2}$ precipitates in $\mathrm{Al}$ alloy [6]. The intensity ratios between SAXS and SANS are $14( \pm 10 \%)$ and $225( \pm 10 \%)$, respectively. The scattering length densities are obtained from mass densities and nominal compositions for matrices and from lattice constants and stoichiometric compositions for precipitates [27]. The expected intensity ratio yields 14.1 for $\mathrm{TiC}$ and 230 for $\mathrm{MgZn}_{2}$. These values coincide with the observed values within the error.

Therefore, we can conclude that iANS can give sufficiently high-quality profiles for quantitative analysis on an absolute unit scale even using CANS and that

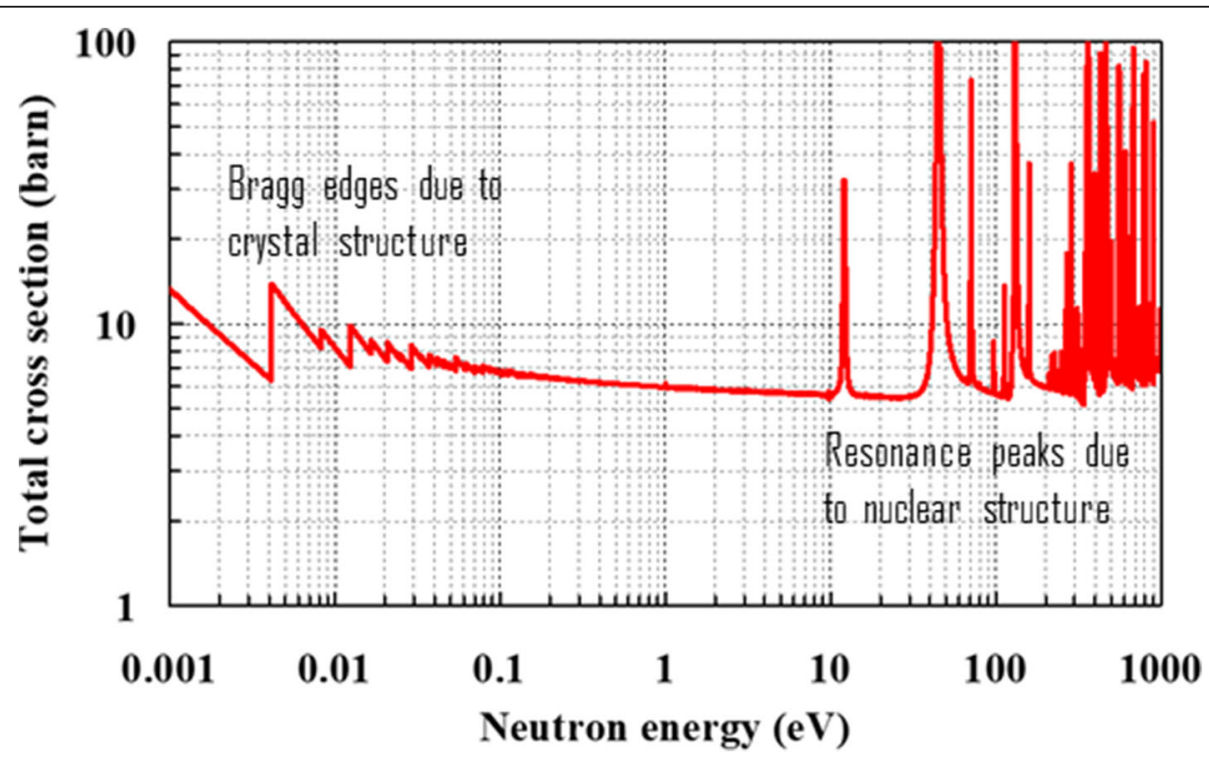

Fig. 8 An example of the neutron cross section 
evaluation of composition and phase only using inhouse SAS instruments is now feasible and ready for further research in various alloy systems.

\subsection{Pulsed neutron imaging}

Neutron imaging has been used in various fields in the world and proved to be very useful. The neutron sources used are from radioisotope neutron sources to big neutron sources. In traditional applications, it uses a white neutron beam, namely a wide energy band beam, and simply gives a transmission contrast image. We found that we could obtain crystallographic information by using an accelerator-driven pulsed neutron source since it gave an energy-dependent transmission spectrum with the use of the time-of-flight method and the spectrum reflected the structure of the neutron cross section including the crystallographic information $[4,5]$. Even at a reactor neutron source, the energy selective imaging has become popular [28]. By using a pulsed source, we can obtain an energy-dependent spectrum continuously, which is a large merit compared with a reactor source.
Figure 8 shows an example of the neutron cross section. We can see a sawtooth-like structure at an energy region less than about few $10 \mathrm{meV}$. Each sharp edge, called as Bragg edge, corresponds to $2 \times d_{h k l}$, where $d_{h k l}$ is the lattice plane spacing of Miller index (hkl) of the crystal structure. The lowest edge corresponds to $2 d_{\max }$ and below this energy the diffraction cannot occur. The peaks that appear at a higher energy region over $10 \mathrm{eV}$ are resonance peaks that energy differs from nucleus to nucleus.

From the low-energy region, the crystallographic information is deduced by using following equations [5]. The neutron transmission $\operatorname{Tr}(\lambda)$ as a function of the neutron wavelength $\lambda$ is written as

$$
\operatorname{Tr}(\lambda)=\exp \left(-\sigma_{\text {tot }}(\lambda) \rho t\right)
$$

Here, $\sigma_{\text {tot }}(\lambda)$ is the total cross section, $\rho$ the atomic number density, and $t$ the thickness of a sample material. The total cross section consists of elastic coherent scattering $\left(\sigma_{\text {coh }}^{\text {ela }}\right)$, elastic incoherent scattering $\left(\sigma_{\text {incoh }}^{\text {ela }}\right)$, in-
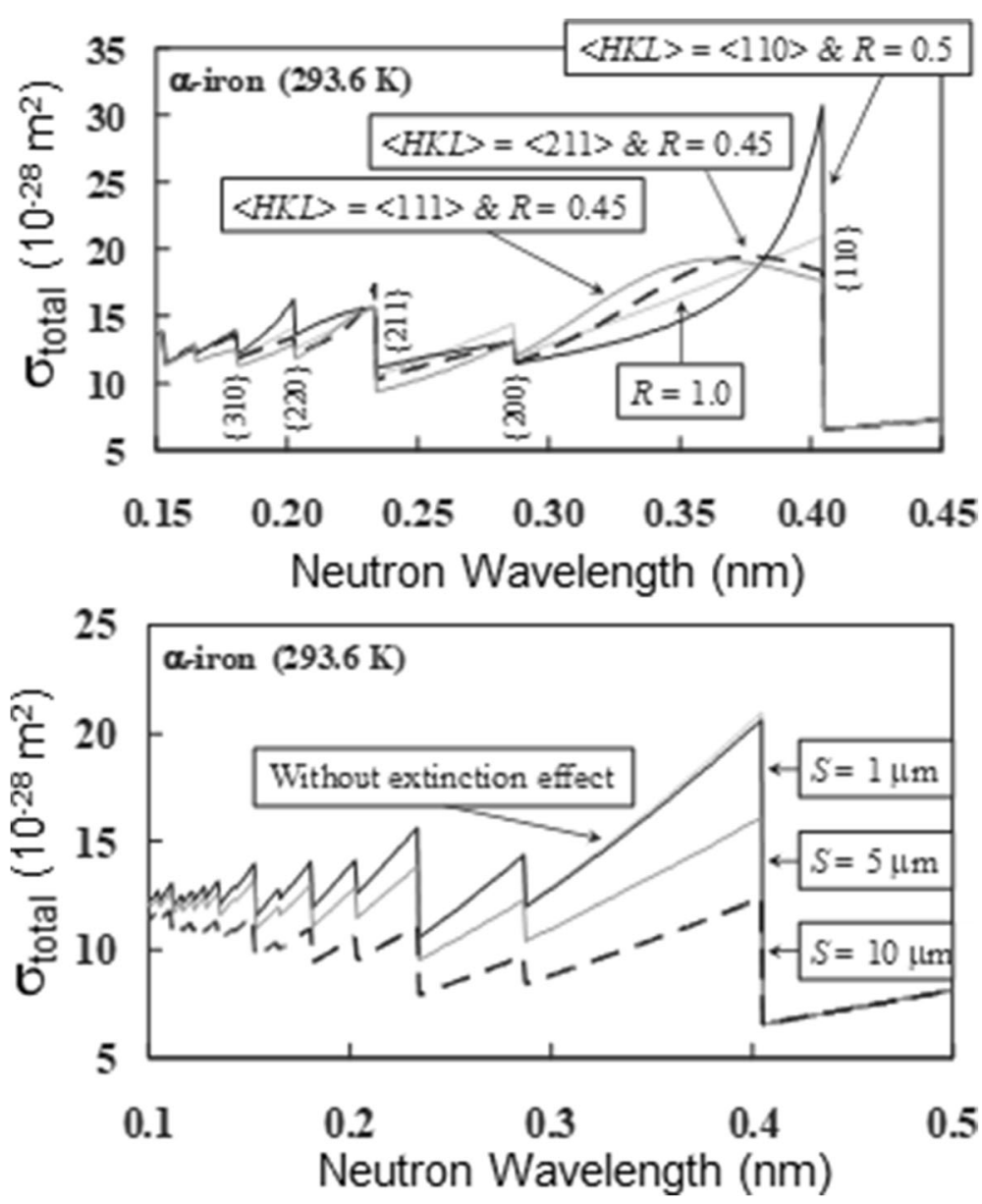

Fig. 9 Cross-section change due to (a) anisotropy and (b) crystallite size [5] 
elastic coherent scattering $\left(\sigma_{\text {coh }}^{\text {inela }}\right)$, inelastic incoherent scattering $\left(\sigma_{\text {incoh }}^{\text {inela }}\right)$, and absorption cross section $\left(\sigma_{\text {abs }}\right)$, as indicated below:

$$
\begin{aligned}
\sigma_{\text {tot }}(\lambda)= & \sigma_{\text {coh }}^{\text {ela }}(\lambda)+\sigma_{\text {incoh }}^{\text {ela }}(\lambda)+\sigma_{\text {coh }}^{\text {inela }}(\lambda) \\
& +\sigma_{\text {incoh }}^{\text {inela }}(\lambda)+\sigma_{\text {abs }}(\lambda) .
\end{aligned}
$$

The elastic coherent scattering cross section relates directly to Bragg edges. The cross section considering distortion of the crystal structure is indicated by the following equation.

$$
\sigma_{\mathrm{coh}}^{\mathrm{ela}}(\lambda)=\frac{\lambda^{2}}{2 V_{0}} \sum_{h k l}\left|F_{h k l}\right|^{2} d_{h k l} R_{h k l}\left(\lambda-2 d_{h k l}\right) P_{h k l}\left(\lambda, 2 d_{h k l}\right) E_{h k l}\left(\lambda, 2 d_{h k l}, F_{h k l}\right),
$$

where $V_{0}$ is the unit cell volume and $F_{h k l}$ is the crystal structure factor. The first factor $R_{h k l}\left(\lambda-2 d_{h k l}\right)$ is the edge profile function, including the $d_{h k l}$ information. From the $d_{h k l}$, crystal structure is deduced. Shift of the edge position occurs as a result of the strain of the crystal structure. The second factor $P_{h k l}\left(\lambda, 2 d_{h k l}\right)$ presents the anisotropy effect due to the preferred orientation, which changes the whole shape of the Bragg edge transmission spectrum. Figure 9a shows cross-section change due to the anisotropy effect. Here, $R$ is a parameter indicating the degree of the anisotropy and $R=1$ corresponds to the isotropic case. The anisotropy becomes stronger when the $R$ value departs from 1 . In the figure, the effect of different Miller indexes is shown. It is recognized that the shape around the Bragg edge changes differently depending on the plane and $R$. The third factor $E_{h k l}(\lambda$, $\left.2 d_{h k l}, F_{h k l}\right)$ indicates the effect of crystallite size. As shown in Fig. 9b, the shape is similar to each other but the absolute value of the cross section decreases with increasing the crystallite size. This is due to the double reflection in the same crystallite, which is called as

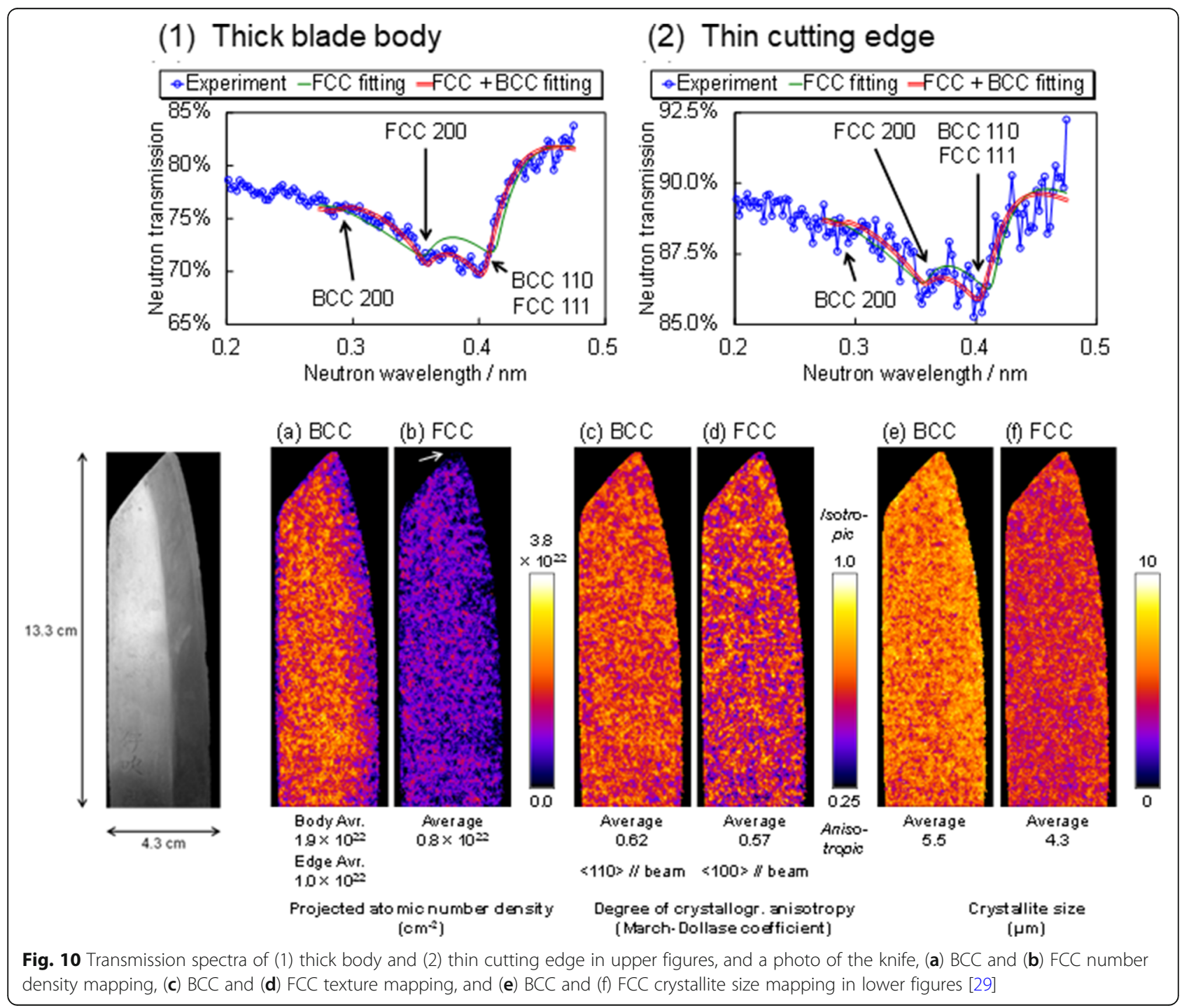


extinction, and the probability increases with the crystallite size.

We applied this method to a kitchen knife "Sekino-magoroku" made in Japan [29]. The knife consists of two crystalline phases of iron: $\alpha$-Fe phase (BCC: body-centered cubic crystal structure) and ( $\alpha$-Fe phase $)+(\gamma$-Fe phase $)$ (FCC: face-centered cubic crystal structure), which were confirmed by X-ray diffraction at both surfaces of the knife. We intended to obtain information on texture (anisotropy), crystallite size, and content of each phase through a thickness direction. Figure 10 shows results of fitting to transmission spectra in (1) and (2) in upper figures, and a photo of the knife, (a, b) number density, (c, d) texture, and (e, f) crystallite size distributions in lower figures. As shown in Fig. 10 (1) and (2), the FCC and $\mathrm{BCC}$ double-phase assumption can give a better fitting curve. Figure 10 (a) and (b) indicate the $\alpha$-Fe thickness becomes thinner around the cutting edge. On the other hand, the $\gamma$-Fe thickness is unchanged over the whole region. At the blade body region, the thickness of $\gamma$-Fe is almost a half $\left(0.8 \times 10^{22} \mathrm{~cm}^{-2}\right)$ of that of $\alpha$-Fe $\left(1.9 \times 10^{22} \mathrm{~cm}^{-2}\right)$. At the cutting-edge region, the thicknesses of both phases are almost the same $\left(\sim 0.9 \times 10^{22} \mathrm{~cm}^{-2}\right)$. It indicates that the knife has a layered structure with the same thicknesses of an $\alpha$-Fe layer and a mixed $\alpha$-Fe and $\gamma$-Fe layer. At the cutting-edge area, only the second layer appears. This result is consistent with the diffraction data and is the unique result of neutrons since the $\mathrm{X}$-ray gives surface information only. Figure 10 (c) and (d) show the texture information. The texture is uniform for both phases and the difference is small between them. Figure 10 (e) and (f) show the crystallite size information and again they are uniform although the size in $\alpha$-Fe (BCC) is a little bit larger than $\gamma$-Fe (FCC). This suggests that this knife did not receive heat treatment and strain hardening around the cutting-edge during the making process.

As another type research, we would like to introduce a study on a Li-ion battery (LIB) as an industrial application [30]. The structure change of a graphite anode in a LIB during the LIB charge-discharge cycle affects the performance of the battery. While charging a LIB electrically, the crystal lattice spacing of graphite $\{002\}$ becomes larger than the discharged one, because the lithium ions intercalate into the graphite layers. We considered the lattice spacing of graphite could be related to the charge level of the LIB and a distribution of charge level would be obtained by Bragg edge transmission. We performed experiments after solving the difficulties due to the large scattering effect of hydrogen and large absorption cross section of $\mathrm{Li}$ that reduces the transmission intensity very much. Figure 11 shows transmission spectra at different charge currents from 0 to $3919 \mathrm{mAh}$. $\{002\}$ peak was clearly observed and the peak moves to a longer wavelength and is blurred with increasing the charge current. Figure 12 shows a photo of LIB and

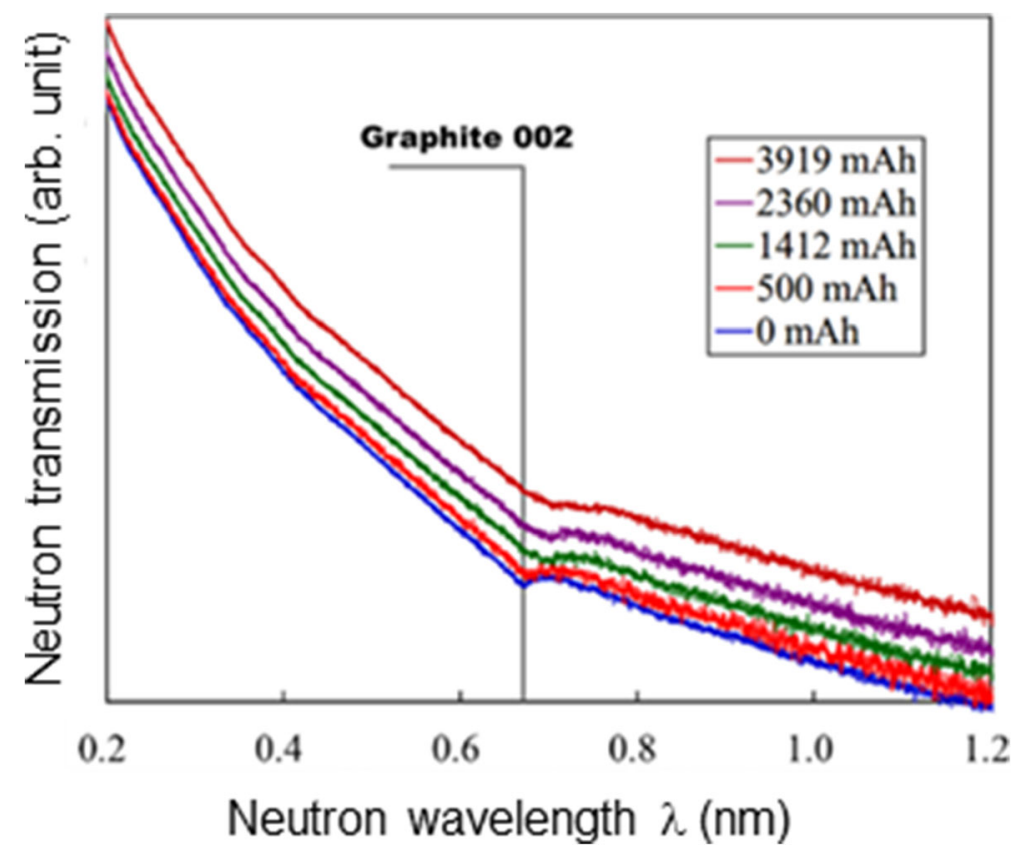

Fig. 11 Transmission spectra at different charge currents from 0 to 3919 mA [30] 


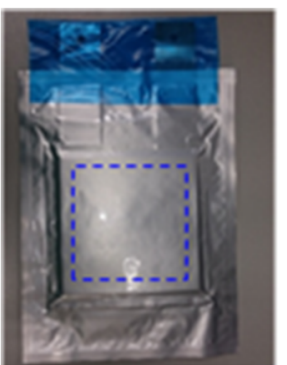

$1412 \mathrm{mAh}$
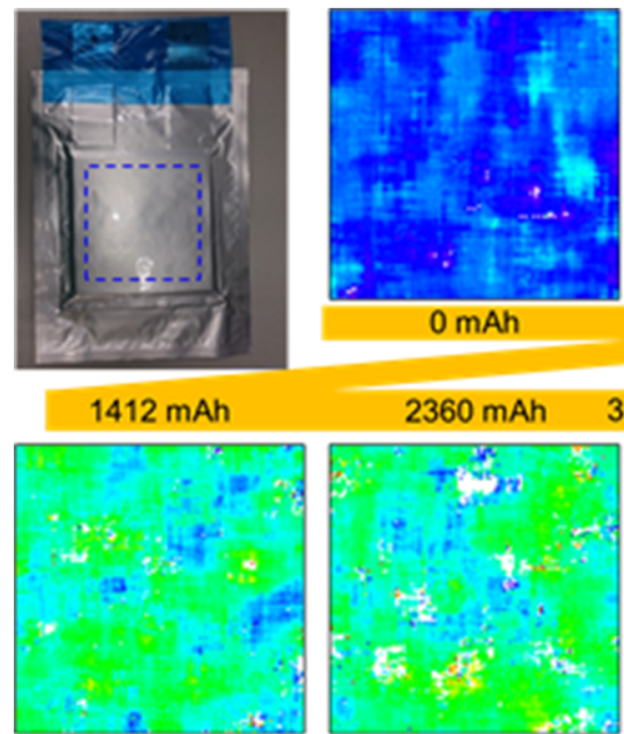

mAh

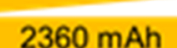

3915

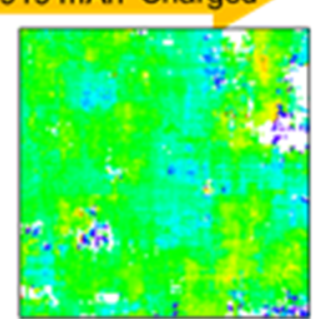

Inter-layer spacing $d_{002}[\mathrm{~nm}]$

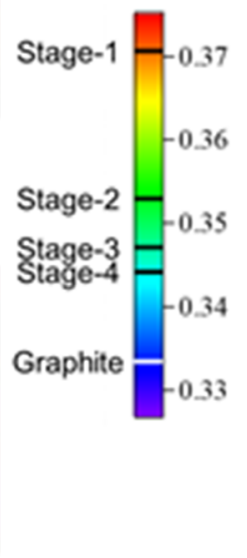

Fig. 12 Photo of LIB and distribution of stages deduced by $\{002\}$ lattice plane spacing [30]

distribution of stages deduced by $\{002\}$ lattice plane spacing. The area size indicated is $9 \mathrm{~cm} \times 9 \mathrm{~cm}$. The average lattice plane spacings of (002) are 0.3354 (graphite), 0.3442 (stage-4), 0.3471 (stage-3), $0.3530 \quad$ (stage-2 $\left(\mathrm{LiC}_{12}\right)$ ), and 0.3706 (stage- $\left.1\left(\mathrm{LiC}_{6}\right)\right) \mathrm{nm}$. With decreasing the stage number, lithium content increases and then the lattice becomes wider. From the results, the ratio of each stage was deduced quantitatively. At the charge level of $0 \mathrm{mAh}$, the negative electrode of graphite is almost in the state of the normal graphite. A little amount of the stage- 4 exists there, which will be due to the production process of the LIB. With the increase in the charge level, the stage- 4 increases initially and then decreasing. The stage- 3 shows a similar tendency. The stage-2 continuously increases from the first of the charge. On the other hand, the stage- 1 rises at the end of the charge. At the last stage of the LIB charge, the charging voltage shows rapid increase and becomes difficult to charge. Even at this charge level, the stage-1 is minor. This means the achievement of the ideal charge
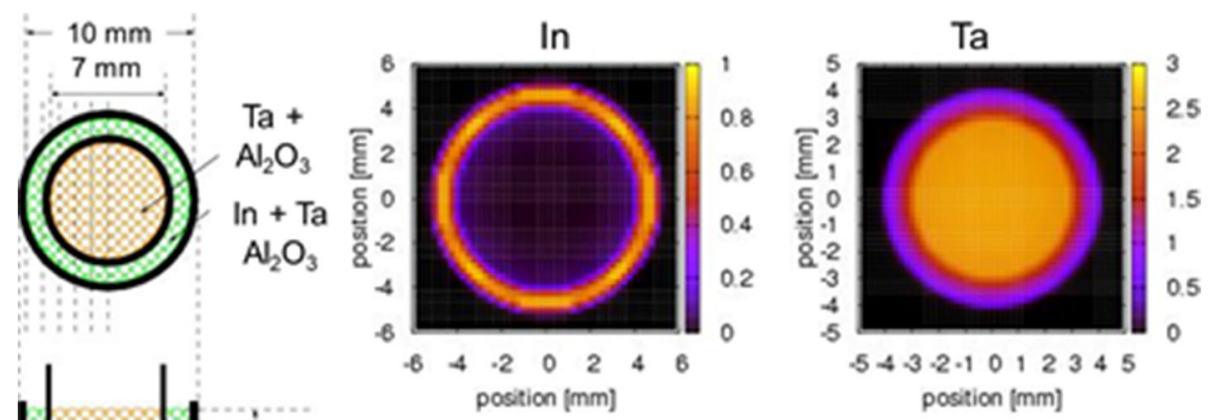

Temperature
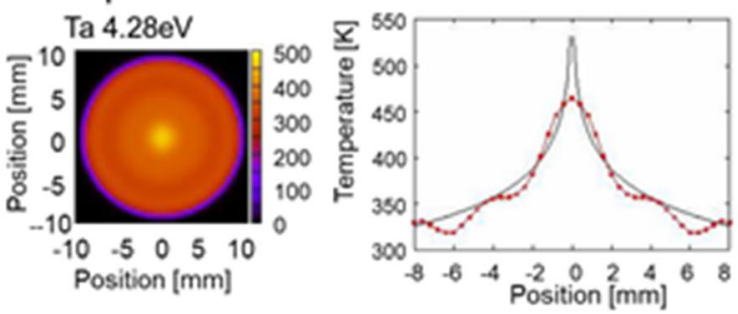

Fig. 13 Example of the resonance imaging [31] 
level is difficult, and the stage-2 structure is considered more stable in the high charge level.

Furthermore, we can deduce nucleus and temperature information by analyzing the resonance peaks that appeared at a higher energy region usually over few $\mathrm{eV}$. The energy of each peak reflects the nuclear structure of each nucleus. Therefore, the nucleus can be identified by analyzing the resonance peaks. Temperature can be measured without contact by using the Doppler broadening of the resonance peak. Figure 13 shows an example of the resonance imaging [31]. The sample is indicated at the left side and its structure is a double cylinder that included a mixture of Ta and In outside and Ta inside with aluminum oxide. Upper figures are tomographic images of In and Ta nucleus distributions. In exists in an outside shell and Ta distributes both inside and outside. The temperature was measured when heating the sample with a heater inserted at the center of the sample. The temperature distribution was shown as a tomographic image at the lower left and as onedimensional distribution at the lower right. The observed temperature curve waves. This is due to insufficient reconstruction of computer tomography since the number of the measuring points was not enough. However, it is indicated that the nucleus distribution and temperature can be measured with this method.

\subsection{Acceleration experiment of soft error}

Digital technologies are more and more becoming important and popular now [32, 33]. However, there are random phenomena called bit errors in semiconductor devices such as in large-scale integrated circuits (LSIs) including memory chips. A soft error is a temporary bit error in a semiconductor. Such errors are caused by a single event upset (SEU) due to unexpected charged particles, which can be produced as secondary ionizing particles resulting from nuclear reactions mainly between a silicon nucleus and a neutron $[34,35]$. Neutrons are produced mainly by proton spallation reactions in the atmosphere. Therefore, neutron soft error mitigation is crucial for ensuring reliability and safety, especially in medical devices, automobiles, airplanes, trains, and core information and communication network equipment for telephones and the Internet.

However, because neutron-induced soft errors are somewhat rare events, it has been difficult to experimentally verify failure processing for these types of malfunctions. To overcome this difficulty, acceleration tests using artificial intense neutron sources have been so far carried out at relatively high-energy particle accelerator facilities producing neutrons with a spectrum similar to the terrestrial neutron spectrum, e.g., TNF at TRIUMF in Canada [36], LANSCE at LANL in the USA [37], ISIS

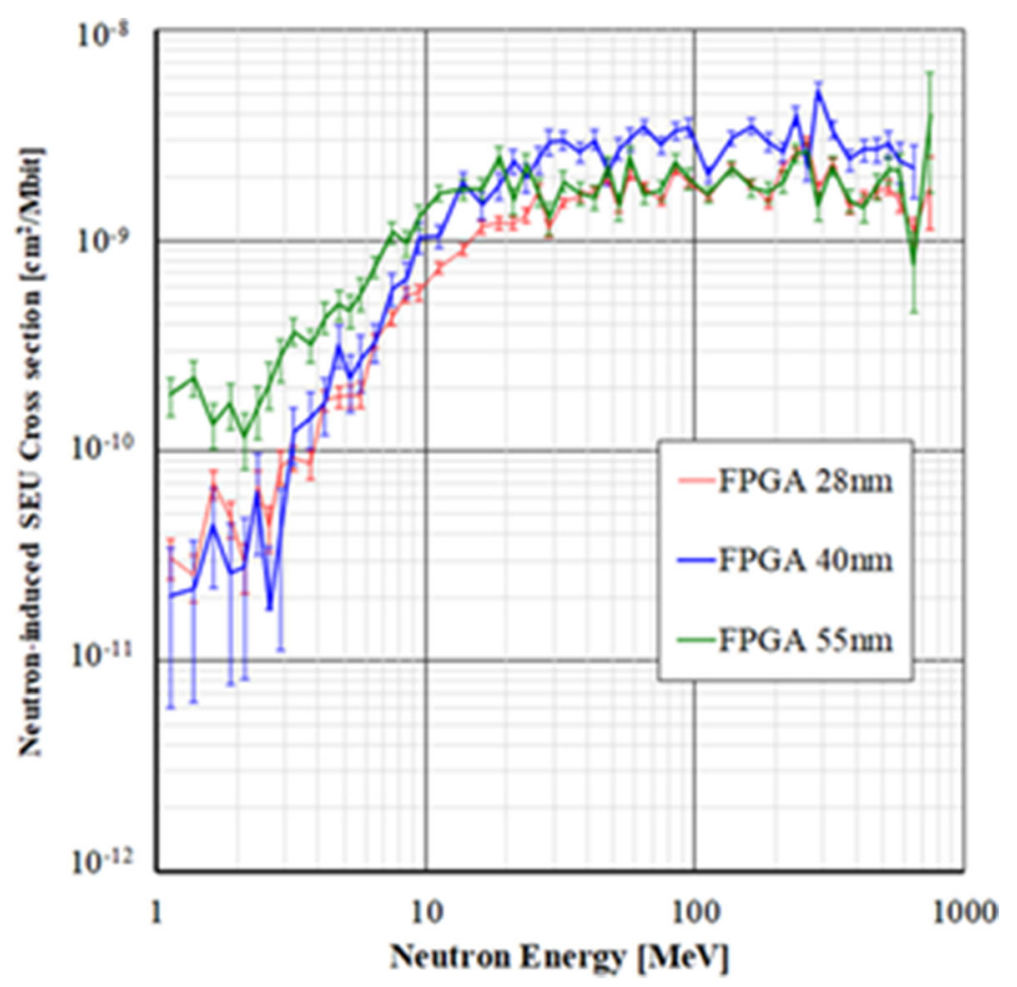

Fig. 14 Neutron-induced SEU cross sections [43] 


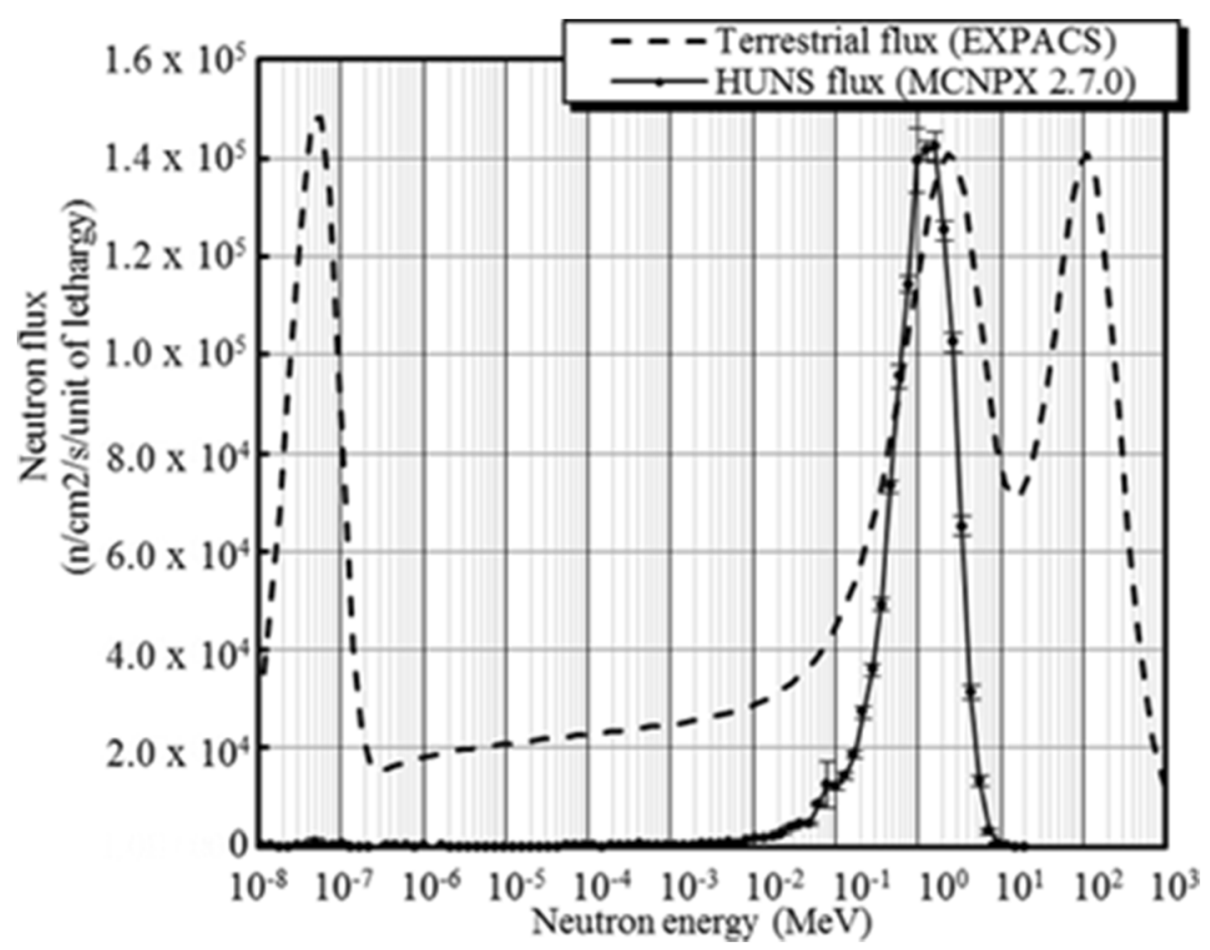

Fig. 15 Comparison of simulated spectral neutron flux data for the HUNS facility (solid line and symbols) and for the natural terrestrial environment at Tokyo, Japan, at sea level (dashed line) [42]

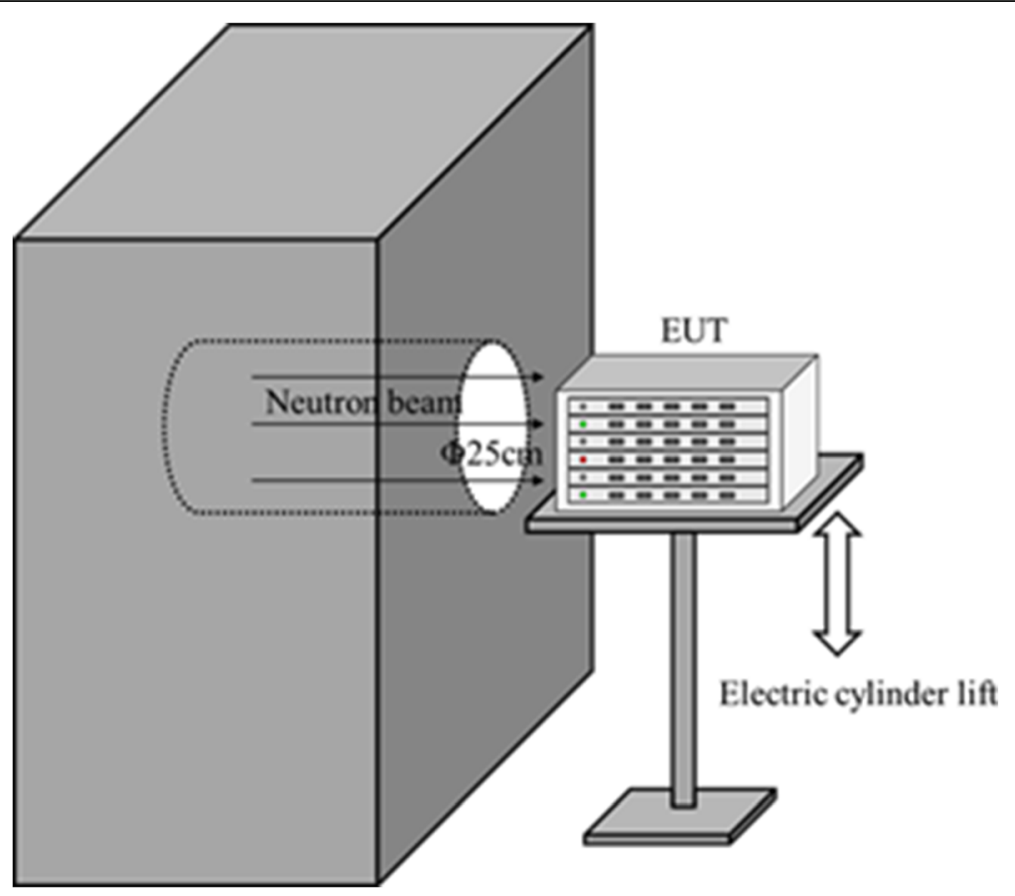

Fig. 16 Schematic view of the experimental setup [42] 


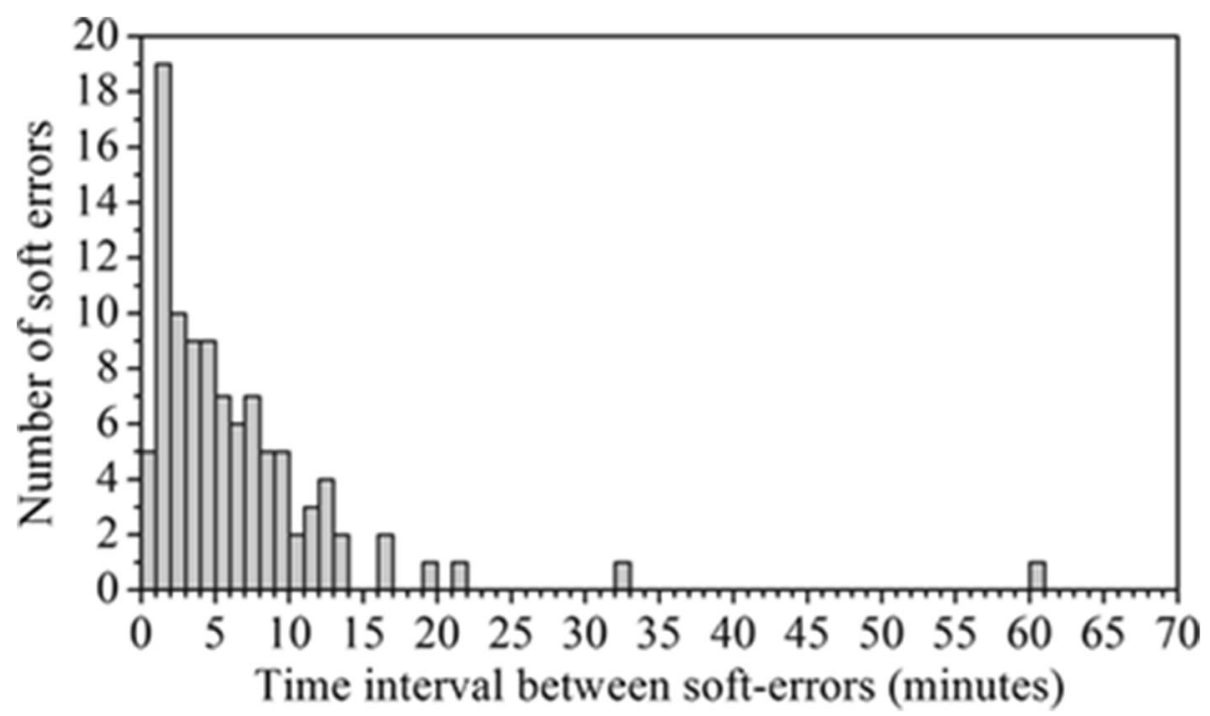

Fig. 17 Time intervals between two soft errors [42]

at RAL in the UK [38], ANITA at TSL in Sweden [39], ISNP/GNEIS in Russia [40], and RCNP at Osaka University in Japan [41].

The major reactions are neutron-Si reactions. They are indicated below with the threshold energy Es [42]. ( ) is the content of each isotope.
${ }^{28} \mathrm{Si}(92.23 \%): \mathrm{Es}=4.00 \mathrm{MeV}$ for $(\mathrm{n}, \mathrm{p})$ and $\mathrm{Es}=2.75 \mathrm{MeV}$ for $(\mathrm{n}, \alpha)$ ${ }^{29} \mathrm{Si}(4.67 \%): \mathrm{Es}=3.01 \mathrm{MeV}$ for $(\mathrm{n}, \mathrm{p})$ and $\mathrm{Es}=0.04 \mathrm{MeV}$ for $(\mathrm{n}, \alpha)$

${ }^{30} \mathrm{Si}(3.1 \%): \mathrm{Es}=8.04 \mathrm{MeV}$ for $(\mathrm{n}, \mathrm{p})$ and $\mathrm{Es}=4.31 \mathrm{MeV}$ for $(\mathrm{n}, \alpha)$

Recently, neutron-induced SEU cross sections were measured as a function of the neutron energy for several

(a) Type A

1st test) FPGA-based device

2nd test) ASIC-based device

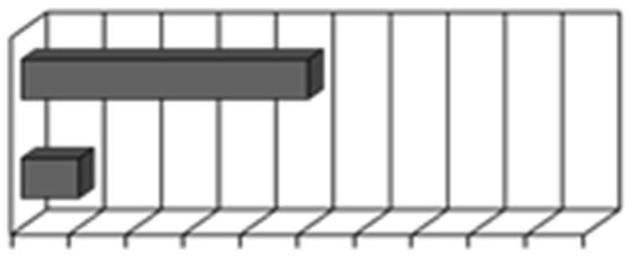

(b) Type B

1st test) Parity function

2nd test) ECC function

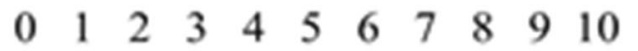

(c) Type C

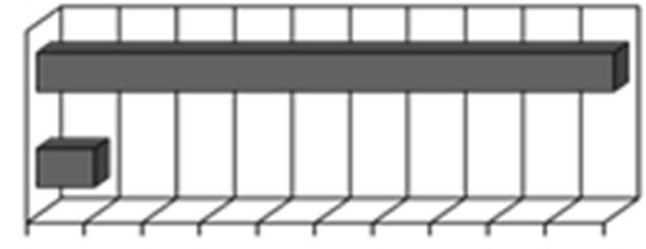

1st test)

w/o auto recovery function

2nd test)

$\mathrm{w} /$ auto recovery function
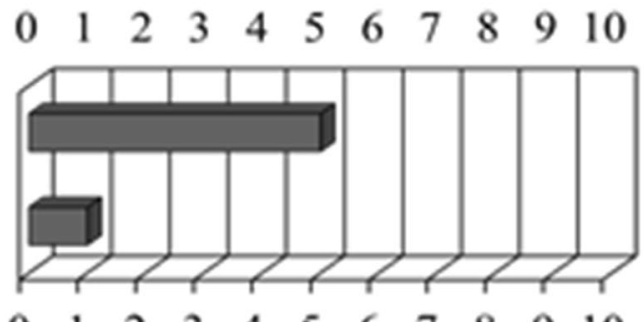

$\begin{array}{llllllllllll}0 & 1 & 2 & 3 & 4 & 5 & 6 & 7 & 8 & 9 & 10\end{array}$

Comparison of neutron soft-error rate

Fig. 18 Comparison of relative error rate for three types of systems before and after applying improving method [42] 
different design rules (minimum processing line width) FPGAs as shown in Fig. 14 [43]. It is recognized from the figure that the cross sections increase from around few $\mathrm{MeV}$ and saturate around $20 \mathrm{MeV}$ for all samples with a design rule of 28,40 , and $55 \mathrm{~nm}$. Therefore, soft errors can be induced by the $\mathrm{MeV}$-range neutrons produced by compact neutron sources and acceleration test is possible. At CANS, trial and error experiments are easily carried out to improve experimental conditions due to its flexibility and low cost.

The acceleration tests were performed at HUNS [42], which produced neutron intensity is about $10^{12} \mathrm{n} / \mathrm{s}$. Figure 15 shows the comparison of simulated spectral data for the HUNS facility (solid line and symbols) and for the natural terrestrial environment at Tokyo, Japan, at sea level (dashed line). The terrestrial spectrum was multiplied by the factor $1.9 \times 10^{8}$ to compare the flux shapes. The intensity at HUNS was about $3.4 \times 10^{6}$ times higher than the terrestrial spectrum over $5 \mathrm{MeV}$. Figure 16 shows a schematic view of the experimental setup. Here, a whole equipment (Packet Transport Multiplexer (PTM) link system [44]) built into a rack was irradiated, which is called as equipment under test (EUT). Figure 17 shows time intervals between two soft errors, relating to the neutron intensity. The frequency became maximum between 1 and $2 \mathrm{~min}$ and the distribution suggested that the accelerator rate was about $10^{6}$ times compared with the natural terrestrial condition. Furthermore, devices to decrease the soft error rate were examined. Figure 18 shows the comparison of relative error rates for three types of systems. The reduction of the error rate was observed for all systems. One of the most important points of the measurements is that this kind of measurements can be performed at CANS within reasonable time scale and trial and error experiments can be easily executed. In 2018, international standards providing guidelines to protect telecommunication equipment from soft errors caused by particle radiation produced by cosmic rays were adopted by ITU-T (International Telecommunication Union - Telecommunication Standardization Sector), which consist of ITU K.124 (Overview of particle radiation effects on telecommunication systems), ITU K.130 (Neutron irradiation test methods for telecommunication equipment), ITU K.131 (Design methodologies for telecommunication systems applying soft error measures), ITU K.Suppl.11 (Soft error measures for field programmable gate arrays), TU K.139 (Reliability requirements for telecommunication systems affected by particle radiation), and ITU K.138 (Quality estimation methods and application guidelines for mitigation measures based on particle radiation tests) (https://news.itu.int/new-itustandards-to-prevent-soft-errors-caused-by-cosmic-rays/). In ITU K.130, CANS is described as a neutron source for the soft error test.

\subsection{Boron neutron capture therapy}

Boron neutron capture therapy has been performed by using nuclear reactors and their clinical results proved the usefulness of BNCT. The treatment has been applied to brain cancer, head and neck cancer, and skin cancer. However, the number of operating reactors decreased and it was desired to construct the BNCT facility in or near a hospital. Therefore, BNCT facilities have been constructed or under planning based on various accelerators in the world.

Boron neutron capture therapy is a combined therapy of radiation therapy and drug delivery of boron agent. Boron has a high absorption cross section for thermal neutrons and the absorption induces a reaction emitting high-energy ${ }^{4} \mathrm{He}(\alpha)$ and ${ }^{7} \mathrm{Li}$ nucleus. In the treatment, before irradiating neutrons, a boron agent is delivered to cancer cells and then neutrons are irradiated. The boron agent is mainly accumulated in the cancer cells and a ratio of concentration between the normal cells and cancer cells is normally about 3.5 in the case of BPA (Boronophenylalanine: $\quad 4^{10} \mathrm{~B}$-borono- $\mathrm{L}-$ phenylalanin). $\mathrm{BPA}$ is now the most used boron agent and officially approved

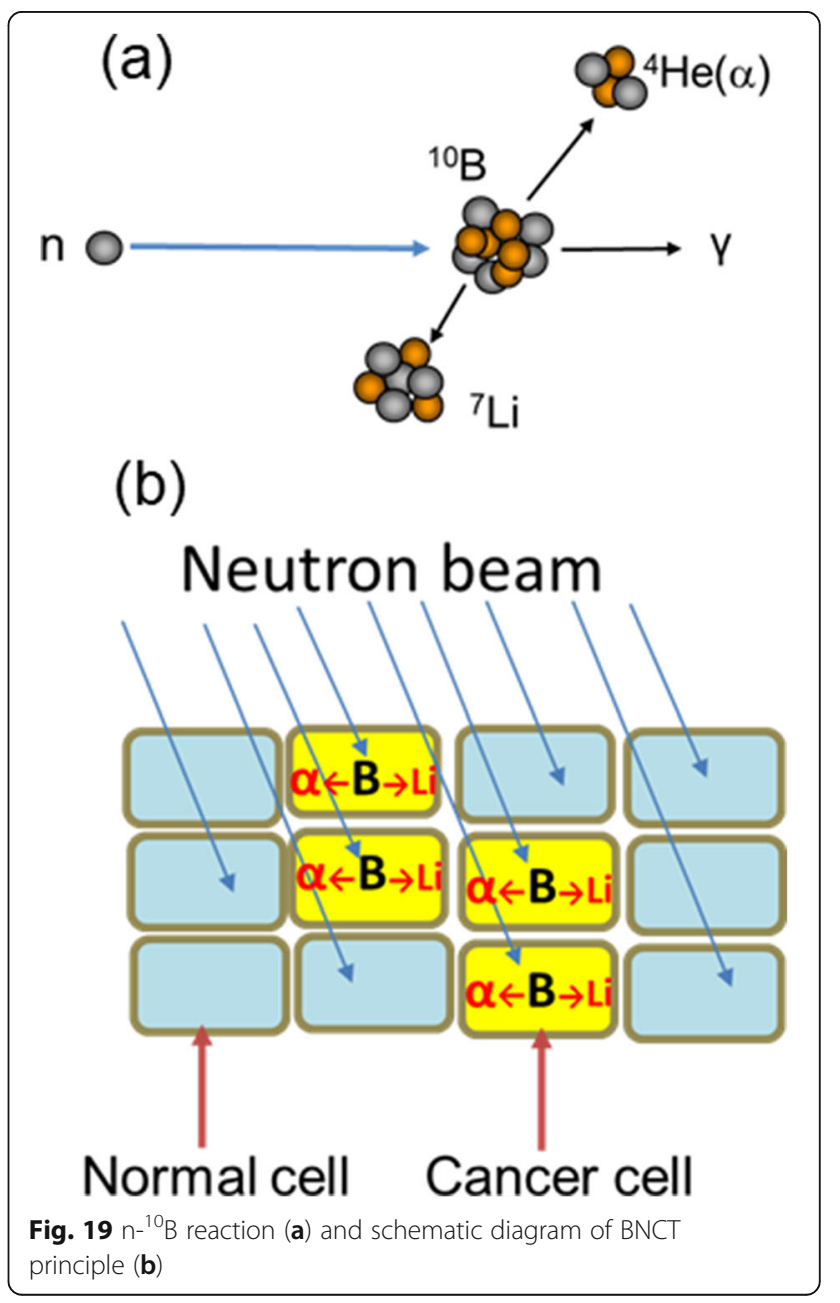


as a BNCT agent in Japan. Figure 19 simply shows BNCT principle: the boron-neutron reaction where two particles are emitted and the reaction in cancer cells.

As shown in the figure (b), $\alpha$ has energy of $1.47 \mathrm{MeV}$ and ${ }^{7} \mathrm{Li}$ has $0.84 \mathrm{MeV}$ and each range is about $9 \mu \mathrm{m}$ and $5 \mu \mathrm{m}$. They have high linear energy transfer (LET): $\alpha$ particle $\approx 150 \mathrm{keV} / \mu \mathrm{m}$ and ${ }^{7} \mathrm{Li} \approx 175 \mathrm{keV} / \mu \mathrm{m}$. These particles provide high energy along their brief pathway that corresponds to an area of a single cell. Therefore, this method in principle kills the cancer cells selectively and the damage of the normal cell will be reduced. The neutrons injected into the human body are epithermal neutrons $(0.5 \mathrm{eV}-10 \mathrm{keV})$ to treat a deep positioned cancer so as to obtain high-intensity flux of thermal neutrons (less than $0.5 \mathrm{eV}$ ) around the cancer due to moderation of epithermal neutrons in the body.

The energy spectrum variation scheme from the target to the human body is shown in Fig. 20. High-energy neutrons produced in a target (Fig. 20 (a)) are moderated in a beam shaping assembly (BSA) to form an epithermal neutron-rich spectrum (Fig. 20 (b)) and the epithermal neutrons are injected into the body, where a thermal neutron spectrum is formed (Fig. 20 (c)). Required characteristics of the BNCT neutron source are different from those for the scattering experiment, since it requires not only the intensity but also low contamination of components like fast and thermal neutrons and photons. The effective neutrons are epithermal neutrons from $0.5 \mathrm{eV}$ to $10 \mathrm{keV}$, which require moderator materials different from the scattering experiment. Fluoride such as $\mathrm{MgF}_{2}, \mathrm{CaF}_{2}$, and $\mathrm{AlF}_{3}$ are usually used as a moderator and a lead reflector is popular [45].

The accelerator-based BNCT system is most intensively developed in Japan. Other major countries are Finland, Russia, Argentina, Israel, Italy, China, and the Republic of Korea. In Japan, there are three most advanced facilities already treating patients: C-BENS at Kyoto University [46], Southern Tohoku Hospital [47], and Kansai BNCT Medical Center (https://www.omp.ac. $\mathrm{jp} / \mathrm{en} / \mathrm{kbmc} . \mathrm{html}$ ), all of which use a $30 \mathrm{MeV}-1 \mathrm{~mA}$ proton cyclotron and Be as a neutron producing target [2]. The first clinical trials in the world were performed [48]. The system obtained approval from the Japanese government for manufacturing and selling as a medical device in 2020. The fourth facility is at the National Cancer Center in Tokyo [49], based on an RFQ linac and the pLi reaction.

The facilities using the ${ }^{9} \mathrm{Be}(\mathrm{p}, \mathrm{n})^{9} \mathrm{~B}$ reaction at lower proton energies exist in Japan and Korea. In Tsukuba in Japan, a large interinstitutional project centered around Tsukuba University is ongoing [50] and is based on a linac with $8 \mathrm{MeV}$ (3 MeV RFQ+ 5MeV DTL) and a designed maximum current of $10 \mathrm{~mA}$. A similar project completed by Dawonsys. Inc. in Korea is in the commissioning phase. It consists of an RFQ + DTL producing a $10-\mathrm{MeV}, 8-\mathrm{mA}$ proton beam on a thick Be target. There

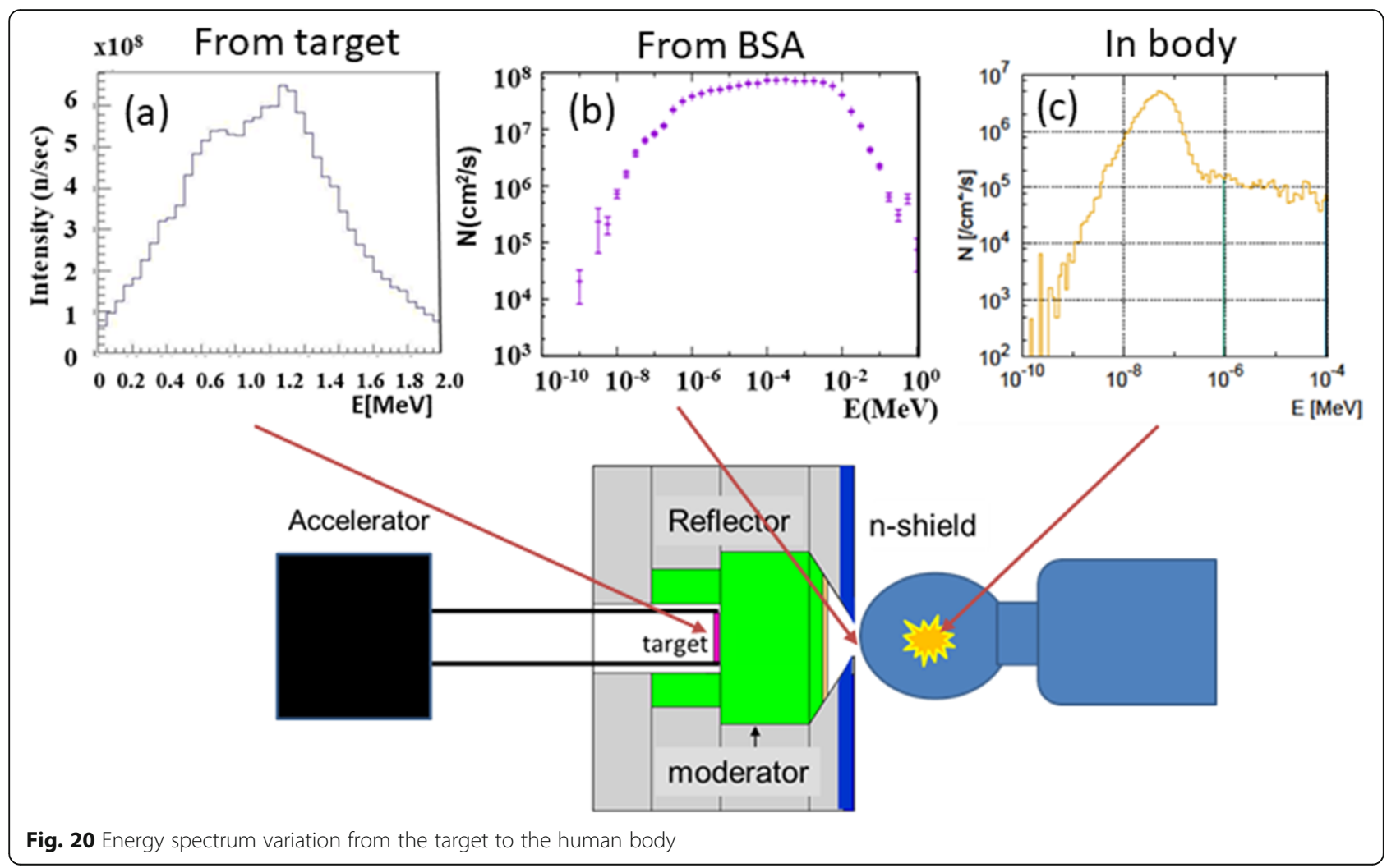


are several ongoing projects based on radiofrequency machines. One is at the Laboratori Nazionali de Legnaro (LNL) in Italy, which is based on a high-intensity RFQ + DTL operating at $5 \mathrm{MeV}$ proton energy and $30 \mathrm{~mA}$ with the ${ }^{9} \mathrm{Be}(\mathrm{p}, \mathrm{xn})$ reaction [51]. The other unique one is at the Soreq research center in Israel and is based on a superconducting RFQ + DTL combined with a liquid Li target [52].

BNCT facilities based on electrostatic accelerators are developed in various places in the world. The most advanced one is in Finland, which is just before the treatment. It is based on a single-ended DC machine with a Li target (http://www.hus.fi/hus-tietoa/uutishuone/Sivut/ BNCT-sädehoitolaite-HUSiin-ensimmäisenä-maailmassa. aspxospital). Others are in Russia using vacuum insulated tandem [53] and China Xiamen Humanity Hospital using the same machine, Argentina using electrostatic Quadrupole (ESQ) [54], and NUANS at Nagoya University in Japan using Dynamitron [55]. All projects other than Argentina use p-Li reaction and the Argentina project $\mathrm{d}$-Be or $\mathrm{d}-{ }^{13} \mathrm{C}$.

BNCT is becoming more and more popular in the world and several countries, the UK, USA, etc., are considering new projects.

\section{Conclusions}

The neutrons are widely used in various fields and the application area is expanding. Traditional neutron scattering applications are still useful for new material and science studies and also for new industrial applications such as battery development. Not only the traditional instruments but also new methods appeared even in the traditional field by using the compact accelerator-driven neutron sources. Soft error acceleration test is an up-todate and important field for the compact neutron sources to promote the development of new high resistive devices and to ensure less trouble society about electric devices used in various fields. BNCT is one of the hopeful fields to give a chance to treat cancers so far not well cured.

To fulfill the requirements for the neutron source for future applications, a more compact neutron source with a required intensity is still desired.

\section{Acknowledgements}

Not applicable

\section{Code availability}

Not applicable

\section{Author's contributions}

All the document was written by Y.K. The author read and approved the final manuscript.

\section{Funding}

Not applicable
Availability of data and materials

Not applicable

\section{Declarations}

Ethics approval and consent to participate

Not applicable

Consent for publication

Not applicable

\section{Competing interests}

The author declares that there are no competing interests.

Received: 17 June 2021 Accepted: 28 July 2021

Published online: 07 September 2021

\section{References}

1. Neutron scattering facilities in Europe, ESFRI Scripta: Vol. I, (2016). https:// www.esfri.eu/sites/default/files/NGL_CombinedReport_230816_Complete\%2 0document 0209-1.pdf

2. Y. Kiyanagi, Y. Sakurai, H. Kumada, H. Tanaka, Status of accelerator-based BNCT projects worldwide, AIP Conf. Proc. 2160, 050012 (2019). https://doi. org/10.1063/1.5127704

3. I.S. Anderson, C. Andreani, J.M. Carpenter, G. Festa, G. Gorini, C.-K. Loong, R. Senesi, Research opportunities with compact accelerator-driven neutron sources. Phy. Rep. 654, 1 (2016). https://doi.org/10.1016/j.physrep.2016.07.007

4. Y. Kiyanagi, T. Kamiyama, H. Iwasa, F. Hiraga, Key Eng. Mater. 270-273, 1371 (2004)

5. H. Sato, T. Kamiyama, Y. Kiyanagi, Mater. Trans. 52, 1294 (2011)

6. T. Ishida, M. Ohnuma, B.S. Seong, M. Furusaka, ISIJ Int. 57, 1831 (2017)

7. K. Nedunchezhian, N. Aswath, M. Thiruppathy, S. Thirugnanamurthy, Clin. Diagn. Res. 10, ZE01-ZE04 (2016). https://doi.org/10.7860/JCDR/2016/19890. 9024

8. H. Iwashita, H. Sato, H. Arai, T. Kotanigawa, K. Kino, T. Kamiyama, F. Hiraga, K. Koda, M. Furusaka and Y. Kiyanagi, IEEE Trans. Nucl. Sci., I 64, 689 (2017).

9. International Atomic Energy Agency. Field Estimation of Soil Water Content, Training Course Series No. 30, IAEA, Vienna (2008).

10. C.W. Tittle, Geophysics 26, 27-39 (1961)

11. idem, Nuclear Geophysics, 2, 95-103 (1988).

12. T. Gutberlet, U. Rücker, E. Mauerhofer, P. Zakalek, T. Cronert, J. Voigt, J. Baggemann, J. Li, P. Doege, S. Böhm, M. Rimmler, O. Felden, R. Gebel, O. Meusel, H. Podlech, W. Barth, T. Brückel, Neutron News 31, 37-43 (2020)

13. F. Ott, A. Menelle, C. Alba-Simionesco, EPJ Web of Conferences 231, 01004 (2020). https://doi.org/10.1051/epjconf/202023101004

14. K.-N. Leung, Nucl. Technol. 206, 1607 (2020). https://doi.org/10.1080/002954 50.2020 .1719800

15. V.N. Kononov, M.V. Bokhovko, O.E. Kononov, et al., Nucl. Instrum. Methods. Phys. Res. A564, 525 (2006). https://doi.org/10.1016/..nima.2006.03.043

16. M.R. Hawkesvorth, At. Energy, Rev. Mod. Phys. 15, 169 (1997)

17. K. Hirota, H. M. Shimizu, Y. Iwashita and F. Naitou, J. the Particle Accelerator Society of Japan, 15, 69-72 (2018).

18. M. Furusaka, H. Sato, T. Kamiyama, M. Ohnuma, Y. Kiyanagi, Phys. procedia, 60, 167-174 (2014)

19. Y. Otake, Plasma Fusion Res., 13, 2401017-1-4 (2018)

20. X. Wang, Q. Xing, S. Zheng, Y. Yang, H. Gong, Y. Xiao, H. Wu, X. Guan, T. Du, Il Nuovo Cimento, 38 C, 185 (2015). https://doi.org/10.1393/ncc/i2015-15185-y

21. Z. Guo, Y. Lu, Y. Zou, K. Zhu, S. Peng, J. Zhao, S. Gao, W. Wen, H. Li, Q. Zhou, H. Ren, P. Lü, H. Zeng, S. Wang, G. Tang, D. Mo, Z. Yuan, D. Xie, X. Yan, J. Che, Phys. Procedia 43, 79-85 (2020)

22. K. Kino, T. Fujiwara, M. Furusaka, N. Hayashizaki, H. Kato, R. Kuroda, K. Michishio, T. Muroga, H. Ogawa, B.E. O'Rourke, N. Oshima, D. Satoh, N. Sei, T. Shishido, R. Suzuki, M. Tanaka, Y. Tomota, H. Toyokawa, A. Watazu, K. Furukawa, K. Nigorikawa, T. Obina, EPJ Web Conf. 231, 01002 (2020). https:// doi.org/10.1051/epjconf/202023101002

23. Y. Oba, S. Koppoju, M. Ohnuma, T. Murakami, H. Hatano, K. Sasakawa, A. Kitahara, J. Suzuki, ISIJ Int. 51, 1852 (2011)

24. M. Ohnuma, J. Suzuki, S. Ohtsuka, S.-W. Kim, T. Kaito, M. Inoue, H. Kitazawa, Acta Mater. 57, 5571 (2009)

25. J. Coakley, B.S. Seong, D. Dye, M. Ohnuma, Philos. Mag. Lett. 97, 83 (2017) 
26. M. Ohnuma, J. Suzuki, S. Ohtsuka, S.-W. Kim, T. Kaito, M. Inoue, H. Kitazawa, Acta Mater. 57, 5571-5581 (2009)

27. A.J. Allen, D. Gavillet, J.R. Weertman, Acta Metall. Mater. 41, 1869 (1993)

28. S. Peetermans, M. Tamaki, S. Hartmann, A. Kaestner, M. Morgano, E.H. Lehmann, Nucl. Instr. Meth. Phys. Res. A757, 28-32 (2014). https://doi.org/1 0.1016/j.nima.2014.04.033

29. H. Sato, K. Mochiki, K. Tanaka, K. Ishizuka, H. Ishikawa, T. Kamiyama and Y. Kiyanagi, Nucl. Instrum. Methods. Phys. Res., A943, 162501 (2019).

30. T. Kamiyama, Y. Narita, H. Sato, M. Ohnuma, Y. Kiyanagi, Phys. Procedia 88, 27-33 (2017)

31. T. Kamiyama, N. Miyamoto, S. Tomioka, T. Kozaki, Nucl. Instrum. Methods. Phys. Res., A605, 91 (2009).

32. E. Stolterman and A. C. Fors, Inf. Syst. Res. IFIP Int. Fed. Inf. Process., 143, 687692 (2004).

33. S. Nambisan, M. Wright, and M. Feldman, Res. Policy, 48, Art. no. 103773 (2019).

34. P. Hazucha, C. Svensson, IEEE Trans. Nucl. Sci. 47(6), 2586-2594 (2000)

35. E. Ibe, "Terrestrial radiation effects in ULSI devices and electronic systems" John Wiley \& Sons, (2015), p.1-12.

36. E. W. Blackmore, "Development of a large area neutron beam for system testing at TRIUMF", IEEE Radiation Effects Data Workshop, (2009), p.157-160.

37. B.E. Takala, "THE ICE HOUSE - neutron testing leads to more-reliable electronics" Los Alamos science. Number 30, 96-103 (2006)

38. C.D. Frost, S. Ansell, G. Gorini, Reliability Physics Symposium, 2009 IEEE International, 952 (2009)

39. A.V. Prokofiev, E. Passoth, A. Hjalmarsson, M. Majerle, IEEE Trans. Nucl. Sci. 61, 1929 (2014)

40. O. A. Shcherbakov, A. S. Vorobyev, A. M. Gagarski, L. A. Vaishnene, E. M. Ivanov, V. S. Anashin, L. R. Bakirov, and A. E. Koziukov, RADECS'2015 conf., Moscow, Sep.19-23, (2015), paper H-3.

41. Y. Iwamoto, M. Fukuda, Y. Sakamoto, A. Tamii, K. Hatanaka, K. Takahisa, K Nagayama, H. Asai, K. Sugimoto, I. Nashiyama, Nucl. Technology 173, 210217 (2011)

42. H. Iwashita, H. Sato, K. Arai, T. Kotanigawa, K. Kino, T. Kamiyama, F. Hiraga, Y. Kiyanagi, K. Koda, M. Furusaka, IEEE Trans. Nucl. Sci. 64, 689-696 (2017)

43. H. Iwashita, G. Funatsu, H. Sato, T. Kamiyama, M. Furusaka, S.A. Wender, E. Pitcher, Y. Kiyanagi, IEEE Trans Nucl Sci. 67, 2363-2369 (2020)

44. M. Ogawa, H. Iwashita, T. Takashima, T. Matsuda, K. Higuchi, and K. Koda, NTT technical review, vol.13 No.7 (2015), available: https://www.ntt-review. jp/archive/ntttechnical.php?contents=ntr201507ra3.html

45. Y. Kiyanagi, Ther. Radiol. Oncol., 12, $2: 55$ (2018). doi: https://doi.org/10.2103 7/tro.2018.10.05

46. H. Tanaka, Y. Sakurai, M. Suzuki, S. Masunaga, T. Mitsumoto, et al., Appl. Radiat. Isot.,69, 1642-1645 (2011).

47. T. Kato, K. Hirose, H. Tanaka, T. Mitsumoto, T. Motoyanagi, K. Arai, T. Harada, A. Takeuchi, R. Kato, S. Yajima, Y. Takai, Appl. Radiat. Isot. 156, 108961 (2020)

48. K. Hirose, A. Konno, J. Hiratsuka, et al., Radiother. Oncol. 155, 182-187 (2020)

49. S. Nakamura, H. Igaki, H. Okamoto, et al., Physica. Medica. 58, 121-130 (2019) https://doi.org/10.1016/j.ejmp.2019.02.010

50. H. Kumada, S. Tanaka, F. Naito, T. Kurihara, T. Sugimura, H. Sakurai, A Matsumura, T. Sakae, EPJ Web Conf. 231, 01003 (2020). https://doi.org/10.1 051/epjconf/202023101003

51. E. Pisent. et al., Proc. $16^{\text {th }}$ Int. Cong. Neutron Capture Therapy, June 14-19, (2014), Finland. http://ICNCT16.org/, p. 66.

52. S. Halfon, A. Arenshtam, D. Kijel, et al., App. Radiat. Isot. 106, 57 (2015)

53. A.A. Zaboronok, V.A. Byvaltsev, V.V. Kanygin, et al., New Armen. Med. J. 11, 6 (2017)

54. D. Cartelli, M.E. Capoulat, J. Bergueiro, et al., App. Radiat. Isot. 106, 18 (2015)

55. K. Watanabe, S. Yoshihashi, A. Ishikawa, et al., App. Radiat. Isot. 168, 109553 (2020) https://doi.org/10.1016/j.apradiso.2020.109553

\section{Publisher's Note}

Springer Nature remains neutral with regard to jurisdictional claims in published maps and institutional affiliations. 